
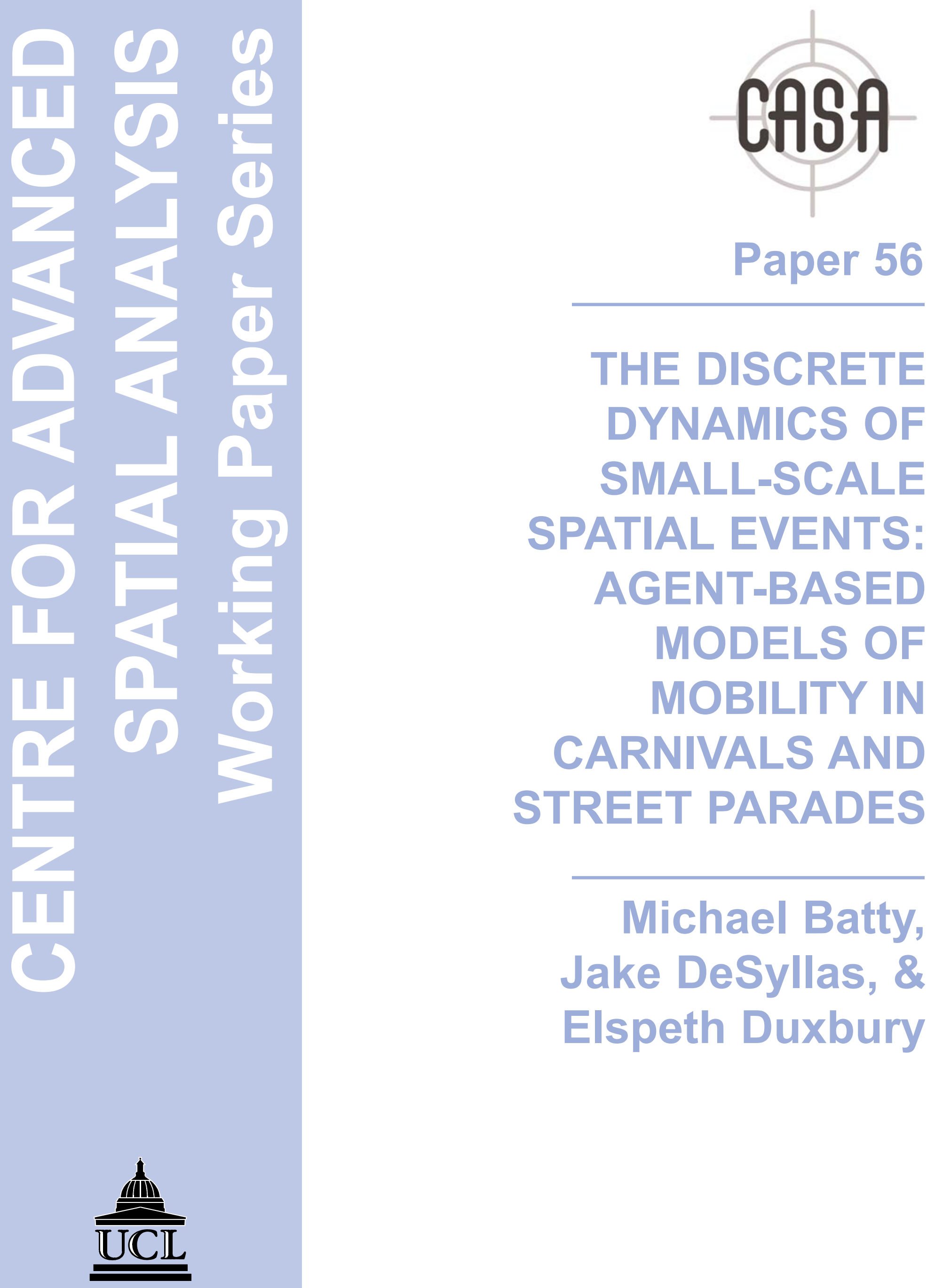

Michael Batty, Jake DeSyllas, \& Elspeth Duxbury 
Centre for Advanced Spatial Analysis

University College London

1-19 Torrington Place

Gower Street

London WC1E 6BT

[t] +44 (0) 2076791782

[f] +44(0) 2078132843

[e] casa@ucl.ac.uk

[w] www.casa.ucl.ac.uk

http//www.casa.ucl.ac.uk/working_papers/paper56.pdf

Date: July 2002

ISSN: 1467-1298

(C) Copyright CASA, UCL

The Discrete Dynamics of Small-Scale Spatial Events: Agent-Based Models of Mobility in Carnivals and Street Parades

Michael Batty*, Jake DeSyllas†, \& Elspeth Duxbury†‡

${ }^{*}$ Centre for Advanced Spatial Analysis, University College London, 1-19 Torrington Place, London WC1E 6BT, UK,

† Intelligent Space Partnership, 68 Great Eastern Street, London EC2A 3JT, UK ¥ Bartlett School of Planning, University College London, 22 Gordon Street, London WC1H 0QB, UK

\{mbatty@geog.ucl.ac.uk, jdesyllas@intelligentspace.com, eduxbury@intelligentspace.com\} 


\title{
The Discrete Dynamics of Small-Scale Spatial Events: Agent-Based Models of Mobility in Carnivals and Street Parades
}

\author{
Michael Batty ${ }^{*}, J^{2}$ e DeSyllas ${ }^{\dagger}, \&$ Elspeth Duxbury ${ }^{\dagger}$
}

10 July 2002

${ }^{*}$ Centre for Advanced Spatial Analysis, University College London, 1-19 Torrington Place, London WC1E 6BT, UK,

$\dagger$ Intelligent Space Partnership, 68 Great Eastern Street, London EC2A 3JT, UK

Bartlett School of Planning, University College London, 22 Gordon Street, London WC1H 0QB, UK

\{mbatty@geog.ucl.ac.uk, jdesyllas@intelligentspace.com, eduxbury@intelligentspace.com\} 


\begin{abstract}
Small-scale spatial events are situations in which elements or objects vary in such a way that temporal dynamics is intrinsic to their representation and explanation. Some of the clearest examples involve local movement from conventional traffic modeling to disaster evacuation where congestion, crowding, panic, and related safety issue are key features of such events. We propose that such events can be simulated using new variants of pedestrian model, which embody ideas about how behavior emerges from the accumulated interactions between small-scale objects. We present a model in which the event space is first explored by agents using 'swarm intelligence'. Armed with information about the space, agents then move in an unobstructed fashion to the event. Congestion and problems over safety are then resolved through introducing controls in an iterative fashion and rerunning the model until a 'safe solution' is reached. The model has been developed to simulate the effect of changing the route of the Notting Hill Carnival, an annual event held in west central London over 2 days in August each year. One of the key issues in using such simulation is how the process of modeling interacts with those who manage and control the event. As such, this changes the nature of the modeling problem from one where control and optimization is external to the model to one where this is intrinsic to the simulation.
\end{abstract}

\title{
Acknowledgments
}

The ESRC Nexsus Project (L326-25-3048) provided partial support for the project. We also wish to thank the Greater London Authority's Carnival Review Committee, Lee Jasper (the Mayor's Advisor), the Metropolitan Police, London Underground Ltd. and the Notting Hill Carnival Trust. 


\section{Introduction: Small-Scale Spatial Events}

Analysis at ever finer geographical scales changes the emphasis from a concern for understanding the structural arrangement of objects to the way in which those objects move to position themselves in space and time. This change in perspective is occasioned by several factors. At finer scales, we become increasingly part of the scene as observers, and begin to identify more closely with elements that are nearer to our everyday experiences. This in turn changes the need to abstract and generalize. Of course, the law of large numbers also breaks down when the phenomena that we observe cannot be classified into categories from which general relationships can be inferred or deduced. But more important is the fact that spaces which are observed at the finest scales cannot be viewed as being predominantly static. What comes into focus is the kind of routine movement which keeps systems energized, alive if you like, and as observers within the scene, our own actions are enough to change the focus of our inquiry from statics to dynamics.

The implications of this are important for geographical information science. As we approach the human scale, relationships begin to be articulated between objects rather than aggregates. Interactions between objects suggest that 'mobility' as well as location is important. More routine processes on finer time scales are introduced and the focus changes from one where the dynamics is abstracted to one where many system elements can be directly observed, often in real-time. Of course aggregate spatial representations are built from the smallest scale where data is collected in realtime but in beginning to represent and model such scales, dynamics becomes essential. This means that very different kinds of spatial events come to our attention, many which have previously been beyond science, indeed beyond geography.

Progress in developing science at this fine scale has been immensely slow for human systems. Systematic data has been absent while ways of defining objects and manipulating them in mathematical and digital environments have only just begun to emerge with the advent of object-orientated representations and agent-based modeling. There has thus been very little work on examining changes in, for example, 
urban form at these scales, despite some extremely promising work on the finegrained socio-economic structure of cities half a century or more ago (Rannells, 1956). As we move to finer scales, new kinds of event which have important spatial manifestations in both the short and long term emerge. For example, the local movement of people in routine contexts clearly affects the location of activities. Changes in the use of land parcels, the continual rebuilding of urban structures, and the changing economy of property are all problems which have barely been touched by systematic science but which now seem to be amenable to deeper explanation.

Perhaps the most obvious problems at the local scale and those that we will illustrate in this paper comprise one-off spatial events which involve the movement of large numbers of people over short periods of time. These largely fall within the sphere of entertainment although some of them relate to work, but all of them involve issues of mobility and interaction between objects or agents which generate non trivial problems of planning, management, and control. The classic example is the football match but rock concerts, street parades, some kinds of shopping, the entry and exit of large numbers of people from high capacity buildings and vehicles such as airports, stations, subway trains, and high buildings, are all examples of such events. Recently disaster scenes involving evacuation have been significant, especially following 9/11 and the war against terrorism where methods for dispersing large concentrations of people are being increasingly explored. These types of event, however, have tended to resist scientific inquiry, and have never been thought to be significant in terms of their impact on spatial structure, or to be worthy of theory. Indeed theories for helping architects design better buildings based on patterns of usage which in turn are dictated by the way people respond and move through small spaces are virtually non-existent, despite occasional but infrequent attempts in the past to provide suitable methods (March and Steadman, 1971).

Much of this is now changing. There is a new momentum to developing geographic information science at much smaller scales which is coming from at least three directions. The most obvious is due to changes in data where ever smaller scales are being observed, measured, and represented digitally. Sensing technology for detecting fine-scale geometries and textures as well as geodemographic data capture through 
electronic transactions are driving the acquisition and measurement of both physical and socio-economic attributes of the small-scale. This is seen for example in the increasing use of GIS software for activities such as urban design (Batty, Dodge, Jiang, and Smith, 1999). The second source revolves around a sea change in the way systems in many fields are currently being conceptualized. There is an increasing recognition that systems must be understood from the bottom-up for many systems function and maintain their structure in this manner. Top-down simulation which usually depends on articulating relationships between system aggregates, is unable to capture the richness in structure and dynamics that many systems display. The way local actions generate emergent structures which have order at more global levels is particularly important to many functioning spatial systems such as the way traffic behaves in networks, the way suburbs and edge cities develop, and the way highly segregated residential areas coalesce within the urban fabric. This is part and parcel of complexity theory which espouses the idea of decentralized functioning and thinking as the basis for new ways of simulation and policy analysis (Johnson, 2001). The third source is a consequence of our growing abilities to interact more globally than hitherto. Consequently, the movement of large numbers of people over short periods of time is an increasing feature of cities and the kinds of spatial problems that they generate are increasingly important.

Before we begin to detail some of these new ways of modeling, we must illustrate in more detail the class of examples that we use here. The events we have in mind are those in which large numbers of people move rapidly to various attractions where the focus of interest is mainly on the attraction and not on others attending the event. Interactions between people, however, do occur through crowding and flocking behavior while the geometry of the environment has an important constraining effect on how people move. Within this nexus, preferences related to the detailed schedules of activities that individuals wish to execute, are embedded within the movement patterns, and these in turn may be tied to fixed locations at the level of buildings, land parcels, and land uses. Such environments may range from those that are not controlled at all as in the case of shopping to those that are highly controlled often through other mobile objects which change the local geometry through barriers, street and building closures, and capacity limits on density. This description of a spatial 
event covers a wide range, from very large concentrations of people at concerts and sports events to movements into local spaces for routine activities of working and shopping through to more drawn out events such as religious festivals, carnivals, and street parades. In this paper, we will illustrate models using the latter examples but the dynamics that we explore are generalizable to other events of this type.

We will begin with a discussion of the local dynamics of small-scale events and ways in which these might be observed and measured. Because such events are subject to considerable control, any model must account for this and we thus propose a two stage structure in which control is gradually introduced into the simulation. In the first stage, we explore the effect of geometry on movement and to this end, we develop an algorithm based on 'swarm intelligence' which generates hierarchies of shortest paths and surfaces of attraction relating the location of events to points at which agents enter the event. In the second stage, agents - visitors/walkers/pedestrians - are launched from the entry points and climb the attraction surface to reach the event. An initial assessment of crowding is then made and if necessary, controls introduced manually to reduce congestion. The model is re-run through this second stage and this process continues until a 'safe solution' is reached.

We then show how the model can be calibrated to data. This model, like many, only touches our knowledge of the event in terms of the data we have. The data requirements are enormous and always less than optimal, quite unlike aggregate modeling where parsimony is the watch word. We develop the model to simulate the effect of changing the route of the parade in the Notting Hill Carnival, an annual event held over 2 days in August each year in a 3.5 square $\mathrm{km}$ area of west central London. This event attracts over 1 million visitors and is widely regarded as posing a major problem of public safety. Our simulations continually refer to such problems of safety and we finally illustrate the way the model can be used to change routes and introduce controls which reduce crowding to acceptable levels. Our focus is not on the application per se but on introducing a generic class of models that might be generalized to a wide variety of small-scale spatial events explicable only through dynamics which involve movement. 


\section{Observing and Understanding Local Dynamics}

Spatial models at any scale imply interactions whose locations are sustained by movements of people, goods, ideas, whatever, between two or more locations which are usually classified as origins and/or destinations. These movements imply processes of interaction over different time scales at different speeds from slow (in years) to fast (in minutes and seconds). At the human scale, interactions occur over different sized areas, each implying a different dynamics, purpose, and goal. Where interactions between people take place in very small spaces of the order of $10 \mathrm{~s}$ of square meters, then the dynamics of movement is dominated by density considerations such as crowding whereas over wider areas of 100 s of square meters or even 10 s of square kilometers, movement is less characterized by geometry, more by cost and purpose. What complicates the dynamics of the small-scale are events in which different scales and types of movement take place with individual movements switching from one purpose and/or scale to another. In our example here, we will deal with movements that take place in confined spaces such as subway stations, along streets where density and crowding are less important, and at fixed attractions where density once again becomes important. But in all these cases, different streams of movement based on different types of individuals form crowds which in turn have their own dynamic.

Although the kinds of events we will simulate appear comparatively simple and occur from fixed origins to destinations like the journey to work, they are quite unlike the journey to work in being much more protracted in duration, with greater freedom of movement in space and time. Such events are complicated by the fact that although the attractions are assumed to be the main foci for such movement, multiple other purposes can intervene and compete such as shopping, eating, and so on. There is also the somewhat mystical property of large crowds being formed with their own momentum which binds them together and drives their movement in very small spaces. Such characteristics are hard to identify, never mind model although such herding instincts due to identity of purpose in the foci of attraction, 'crowd fever' so to speak, are important features. The morphology of such events has barely been 
touched to date. There is little descriptive material at small spatial scales on which good models of these dynamics might be built, and the interpretations that do exist are not found within mainstream geographical, urban, or architectural analysis. There is a useful classification from Canetti (1962) who describes crowds as certain kinds of groups while there is a persistent line of research in psychology from LeBon (1905) on but much of this relates to what Isaac Newton once described as the "madness of crowds', dealing as much with speculation and gambling as with physical concentrations.

Canetti's (1962) classification is however useful. He describes such events as being highly focused on single points of attraction which are spatially associated with agglomerations of individuals. The crowds we are dealing with here form slowly with minimal diversions but because there are competing attractions, there is continual circulation within the highest density places as individuals move to experience adjacent attractions. It is possible in the events simulated here that crowds can grow to sizes and densities which are out of control. Fear and panic can set in as crowds attempt to disperse if densities become too high and safety is compromised. Crowds form at points of ingress and egress where they are channeled into and out of high capacity vehicles like buses, subways trains, and buildings. In short, there is an implicit morphology of crowds which likens them to organically growing and changing phenomena although few have attempted to describe the dynamics and the transformations that take place as crowds form and dissipate. In this context, our events are considerably calmer than crowds at football matches or in Japanese subway trains. Canetti (1962) describes the kind of crowd that we are dealing with here when he says: "There is a .... type of slow crowd which can better be compared with a network of streams. It starts with small rivulets gradually running together. Into the stream thus formed other steams flow, and these, if enough land lies ahead, will in time become a river whose goal is the sea. The pilgrimage to Mecca is perhaps the most impressive example of this slow crowd" (page 40).

We shall see that this picture is close to the way the crowds form in our applications here. It is directly reflected in the dynamics captured within the model but this depends on the way we define the elements or objects that make up the event. It is 
worth thinking of these as mobile agents which move within a geometric landscape based on streets in the urban case. Most models of pedestrian movement are now being developed in this way but here we will not confine our agents simply to the walkers who are visitors at the event. The attractions themselves may be treated as objects, some fixed such as concert venues but others mobile like parades. Ways of controlling the geometry of the landscape - the streets - can also be defined as objects. Barriers and agents such as the police who control access can be mobile while the whole range of emergency services and related vehicles can be similarly considered. Different group types within the crowd might be defined depending upon the variety of attractions and the diversity of the visitors at the event. This variety suggests many different types of interaction between different agent groups - not simply based on human interaction but interaction between the physical landscape and users of that landscape, where the cells that actually define the geometry may themselves be considered as agents (Box, 2001). This type of characterization is similar to that used in particle physics where the concept of the active walker - a particle (agent) that both changes and is changed by its environment (landscape) - has become popular (Schweitzer, 1997).

The critical issue involves the difficulties in observing this kind of system in sufficient detail. Strictly speaking with models which are composed of individuals, there should be data on the decision-making events associated with each individual throughout the time periods and across the space associated with each decision event. All dynamics involves making observations at cross sections in time and interpolating time paths between these sections. This, however, is difficult where actions and interactions are required in real time. Invariably it is not possible as each individual cannot be tagged remotely or directly through human or automated sensors. What is usually possible is good data on the density of crowds but not on paths taken by individuals. This is the same problem that is faced in monitoring vehicular traffic although in that example, movement is constrained to much stricter geometries - the road or rail system - and there is some possibility that the movement of individual vehicles can be tracked. It is difficult to get good path data from closed circuit TV or even from laser scanning data but the idea of tagging pedestrians and monitoring movement this way is probably never going to be possible due to privacy considerations. 
In the case of pedestrian systems, good origin and destination data for each individual is never enough as it is the actual movement through the street system that is important. The best data that has been collected to date is for the most confined spaces where crowding at sports events and in subway stations is under scrutiny. Over wider areas, this has always been problematic and the fact that the best examples are not contemporary indicates the importance of the observation problem (Pushkarev and Zupan, 1975). In fact the lack of path data and data on how crowd and individual types interact will actually influence the kind of models that can be built. As we show here, models need to be built that not only estimate and predict but also enable users to explore existing patterns, predicting possible paths that may be taken in the absence of knowledge about the actual paths taken. Similar problems exist in eliciting preferences which cannot be observed but only inferred through actions and invariably we data on lack behavioral protocols and responses. Agent-based models must therefore be designed to account for such omissions.

\section{The Control of Spatial Events}

Simulation usually follows the conventional cycle of mathematical modeling where data assembly, representation, and analysis is prior to model specification and testing and where prediction is usually only attempted when a good enough fit of the model to reality has been achieved. The final stage of the cycle involves using the model for optimization where model outputs are managed or designed in some way to achieve certain targets or goals. This process works well where models are aggregate and parsimonious, where data is adequate and where it is assumed that the kinds of controls or designs required in the future system are absent from the way the system has evolved in the past. However with small-scale spatial events, these assumptions are no longer tenable. As we have noted, data is always inadequate, by definition, and this puts in doubt the conventional process of model calibration. Moreover many of the events that are of interest in this domain cannot be separated from explicit controls. Many of these are passive, being part of the wider environment, but some of 
them are active in a way that makes them critical to simulation and calibration. For example, as crowds at sporting events get bigger and denser, safety standards come into force through police control of crowd behavior. In the case of crowds which are not policed directly, constraints on the design of physical infrastructure act in a passive way to control behavior.

In short, we cannot build models of spatial events where we assume that data is collected first, the model calibrated, and then if appropriate, used for prediction, thence design and control. What this implies is that the model designed here incorporates all these stages from data assembly to prescription and control and that the process of calibration is contingent on the entire sequence. It is easiest to illustrate this with the example used here. We have some data on where people originate and where they are destined for but we do not have data on their spatial preferences or on the paths that they actually take which connect up their origins to their destinations. Thus the first stage of the model is to generate these paths that are consistent with normal walking behavior. The problem is one of simulating the missing data but in such a way that the most likely behavioral pattern results. Once this is completed, the model can be calibrated in the usual way but as this calibration involves generating missing data, then it must embrace this earlier stage.

However, the event we will be modeling is also highly controlled by the police who channel crowds by closing streets and erecting barriers as well as positioning attractions. These controls are known so we could model the actual situation with these in place but as the purpose of this model is to redesign these controls, then what we actually do is begin our simulation with no controls. We thus assess the situation first in the absence of control, then gradually introduce controls to a level which meets the goals of safety associated with local movements. This means that data, calibration, prediction, and control (prescription) merge into one another; calibration must thus be structured around the whole cycle. When it comes to testing different controls, then the entire cycle must be run again for new controls imply different data patterns which as before are not available and must be generated. In a sense, the kind of model that we have here is in fact a lot closer to the way we know reality is structured than the more parsimonious, aggregate models that have dominated social science to date. 
Control exists despite the general assumption that free markets and unconstrained behavior prevail, while our inability to get inside the system and observe all its attributes means that in any situation, there will always some data that needs to be generated.

There is a further twist to this circularity. The controls themselves cannot be divorced from the event itself, indeed they define the event, and these are also generated from the bottom up. Moreover, these controls cannot be easily modeled without the interventions of those who design them - the police and related authorities in the case of crowding situations. Strictly speaking, the most appropriate way to design and operate this kind of model is to provide an interface to those who actually control the real event and to use their expertise in running the model through its various stages. In the first stage in which data is being assembled, such stakeholder involvement is not required but in the second stage where 'virtual agents' are simulated under different controlled conditions, the way these controls are introduced is best accomplished by the 'real agents' - the police and related authorities - who design these in the first place. As the process of calibration loops around this entire sequence, this breaks the model into stages where different kinds of expertise are required, further complicating the way it needs to be executed. In fact in the experiments which follow, we do not involve the stakeholders in the control phases for we are intent on evaluating existing controls. But in evaluating futures, such involvement is crucial.

Before we explain the class of agent-based models that we consider suited to these types of problem, it is worth a brief review of alternative and complementary approaches. In fact there is a disjunction in the field of pedestrian or walker modeling between models which emphasize density and crowding and those that focus on the way walkers move from origins to destinations. These approaches are not mutually exclusive, they may be complementary but they do not deal with the same kinds of walking phenomena. The former tend to be for confined spaces while the latter for much wider areas; the former are designed for issues of safety and evacuation while the latter are for predicting volumes on different routes between origins and destinations with the assumption that capacity issues will be dealt with naturally. Yet all these models deal with self-organization through push-pull effects which occur 
when individuals form crowds through herding, and when individuals seek to escape from crowds due to panic.

Helbing (1991) and his colleagues have developed a whole series of models which are built around social forces, which relate variously to ideas from fluid flow, particle systems, and flocking (Helbing, Schweitzer, Keltsch, and Molnar, 1997). Similar approaches have been developed by Still (2001), and Hoogendoorn, Bovy and Daamen (2001). Several reviews exist (Helbing, 2001; Helbing, Farkas, Molnar and Vicsek, 2001; and Helbing, Molnar, Farkas, and Bolay, 2001). In contrast there are models being developed by Blue and Adler (2001) usually for more constrained route systems using cellular automata akin to those developed by Nagel and Schreckenberg (1992) for vehicular traffic. These models are also being applied to slightly larger scales by Dijkstra, Jessurun, Timmermans (2002) and Burstedde, Klauck, Schadschneider, and Zittarz (2001). At smaller scales for more ordered flow schedules, queuing models have been adapted but with limited success (Lovas, 1994) while for building and urban spaces where preferences associated with different locations are key, event based simulation has been attempted (Baer, 1974). At the larger scales where movement on malls and even entire neighborhoods is the focus, spatial choice and interaction models have been applied (Borgers and Timmermans, 1986). There have never been enough applications to generalize this field into distinct types for there are elements of each approach in every other. For example, agent based models are now becoming popular at several small scales ranging from town centers (Haklay, Thurstain-Goodwin, O’Sullivan and Schelhorn, 2001) to buildings but there is also the implication that such approaches can be applied at much larger scales (Schweitzer, 1998; Batty, 2001).

\section{Movement Dynamics: A Formal Model}

The best way to illustrate this generic approach is through our example of the Notting Hill Carnival. This event is represented by several groups of agent which move at different speeds - fast, slow, and immobile. We define: walkers $(W)$ who are visitors 
and interact with each other and the Carnival events in real time, paraders $(P)$ who move in more routine fashion along the parade route again in real time, $\underline{\text { bands }}(B)$ which form the fixed sound systems, are immobile but emit noise which decays exponentially from source, thus attracting walkers, and physical objects which we will call streets $(S)$, reflecting building layout and street geometry which act as obstacles to movement but are 'movable' in themselves. In fact, the parade and paraders, the bands, and the street objects can all be 'moved' occasionally but infrequently, and it is these that provide controls which can be manipulated to meet standards of public safety.

Table 1: Varieties and Characteristics of Agent in the Notting Hill Model

\begin{tabular}{|c|c|c|c|c|}
\hline $\begin{array}{l}\text { Agent } \\
\text { Group }\end{array}$ & Agent Type & $\begin{array}{c}\text { Mobility } \\
\text { Level }\end{array}$ & Movability & $\begin{array}{c}\text { Data } \\
\text { Sources }\end{array}$ \\
\hline \multirow[t]{3}{*}{ Walkers $(W)$} & Visiting Parade & Fast in Real Time & $\begin{array}{l}\text { Completely } \\
\text { Flexible }\end{array}$ & \multirow{3}{*}{$\begin{array}{c}\text { Origins and } \\
\text { Destinations } \\
\text { of Observed } \\
\text { Walkers, } \\
\text { Paths not } \\
\text { known }\end{array}$} \\
\hline & Visiting Bands & Fast in Real Time & $\begin{array}{l}\text { Completely } \\
\text { Flexible }\end{array}$ & \\
\hline & $\begin{array}{l}\text { Visiting Bands } \\
\text { and Parades }\end{array}$ & Fast in Real Time & $\begin{array}{l}\text { Completely } \\
\text { Flexible }\end{array}$ & \\
\hline Paraders $(P)$ & Moving Vehicles & $\begin{array}{l}\text { Fast but Fixed } \\
\text { Route }\end{array}$ & $\begin{array}{c}\text { Movable in Long } \\
\text { Term }\end{array}$ & Observed \\
\hline Bands $(B)$ & $\begin{array}{l}\text { Fixed Sound } \\
\text { Systems }\end{array}$ & Fixed & $\begin{array}{c}\text { Movable in Long } \\
\text { Term }\end{array}$ & Observed \\
\hline Streets $(S)$ & $\begin{array}{c}\text { Physical } \\
\text { Objects/Barriers }\end{array}$ & Fixed & $\begin{array}{c}\text { Movable in } \\
\text { Medium Term }\end{array}$ & $\begin{array}{c}\text { Observed and } \\
\text { Managed }\end{array}$ \\
\hline
\end{tabular}

In Table 1 we show these agents in terms of their mobility characteristics and data requirements while in Figure 1, we graph their potential interactions. From these interactions, it is very clear that the focus of the agent structure is on the walkers. These interact with each other through forming crowds by flocking, and then dispersing if congestion is too high. They are directly affected by paraders, street geometry, and sound systems. The paraders and bands interact with themselves in a relatively passive way but not with each other while the street geometry simply affects the walkers. These interactions exists in real time but there are longer term interactions between all agent types: first through changes to the street geometry affecting parades and bands which can occur as control is increased or decreased (the 
broken arcs with no arrows in Figure 1), and second in the much longer term when the parade route and the band locations are themselves changed (the broken arcs with arrows). The self-interactions involve crowding behaviors when visitors come in contact with each other and with the paraders and the bands, while interaction with the street geometry is always in terms of obstacles on movement and behavior. Table 1 also makes clear that at least three kinds of walkers exist in the system: those who interact solely with the bands, those who visit simply to see the parade, and those who visit to engage with both. Although preferences are not explicitly incorporated in this model, these three walker types involve implicit behavioral differences with respect to their attraction to the events that comprise the Carnival.

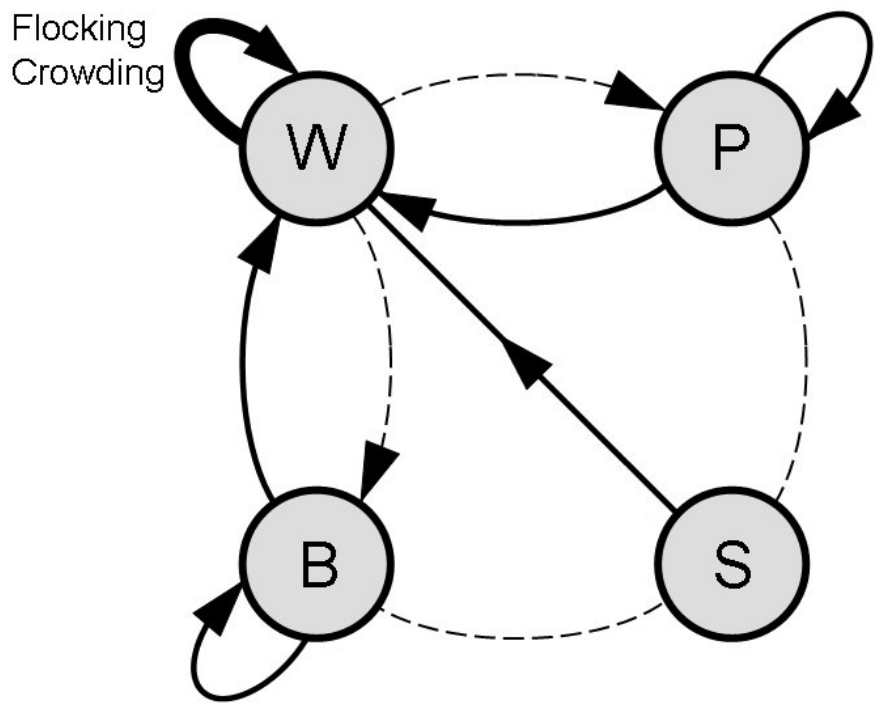

Figure 1: Interactions between Agents

The spatial behavior to be simulated is extremely straightforward. Walkers enter the Carnival area and move through the street system to the various attractions. They interact with each other, the parade, and the sound systems, and these interactions can cause crowding which violates safety limits. The paths they take from the points at which they enter are defined by the noise that they hear, and by feedback from other walkers which leads to 'flocking'. When large crowds form at the various attractions, they disperse if congestion exceeds certain thresholds. If they cannot disperse sufficiently quickly due to the build up, then panic can set in and accidents occur. In terms of the simulation, the paths that walkers take are unknown and must be 
generated and thus the first stage of the model involves generating this 'missing data'. Hierarchies of shortest routes and related accessibility surfaces will mark this stage and these are used in second stage to effect the simulation of walkers from which patterns of crowding and public safety levels can be evaluated. Constraints designed to raise levels of safety are then systematically introduced through a process of optimization-control-management and this will necessitate the two stage model being reiterated until acceptable limits are reached.

To generate the shortest routes, we can either begin with walkers at known origins which are the entry points or with walkers at known destinations, searching either for destinations from origins or vice versa. We will use an algorithm in which all walkers begin to search for the relevant locations randomly but as these are discovered, others simply learn by watching where the successful walkers are moving to. This kind of algorithm is based on a class of behaviors called 'swarm intelligence' (Kennedy, Eberhart, and Shui, 2001). Based on intelligence amongst insects which is determined in a bottom-up fashion through such learning, these techniques are finding wide applicability in all kinds of routing problems from telecommunications to robot manipulation (Bonabeau, Dorigo, and Theraulaz, 1999). The walkers move out randomly from their starting points (which in fact are their ultimate destinations). Those closest to entry points will discover these first and once this occurs, they head back to the starting points which they remember. However to remind themselves of the path between their entry point and starting point, they make a mark by laying a trail. In analogy to the way ants react to the discovery of food sources, they drop 'pheromone'. (Camazine et al., 2001). Other agents who have not yet discovered any destination points see these trails by sensing the scent and this leads them more and more quickly to the various entry points. This process turns out to be one of finding a hierarchy of shortest routes between destinations and entry points. Once an agent has returned to the destination after such a discovery, it begins again but this time reacting to the pheromone surface where it exists. In this way, the hierarchy of routes is reinforced with the shortest being the most heavily trafficked. This first stage is illustrated in Figure 2 with the process of walking during this stage only finishing when the hierarchy begins to converge to a stable set of routes. 
In the second stage, we use the information discovered at the first to construct appropriate accessibility surfaces for each class of walker. Essentially these surfaces links origins (entry points) to destinations (Carnival events), walkers being launched from entry points and 'climbing' these surfaces to reach the attractions. The walkers interact with each other through watching where others walk, thus 'following the crowd.' Walkers flock to the parade routes and to the fixed sound systems and in doing so generate congestion which is resolved by dispersal. Flocking is positive feedback and dispersal is negative. The interaction with the parade can lead to conflict other than congestion for as walkers try to cross the parade route, then accidents can occur. However the greatest source of potential safety problems occurs when crowds build up and are unable to disperse. Dispersal takes time and meanwhile flocking continues especially in areas where the street geometry is highly constrained. As the walkers are launched slowly in a stream from their origins, then it also takes time before they all reach the Carnival area and before a steady state distribution emerges.

Only when this steady state emerges which is sensed when average distances traveled in each time period converge, are we able to make a clear assessment of crowding. In short we simulate the steady state at the peak period during the Carnival and to reach this point in time, we do not simulate all walkers throughout the two-day period but build up the relevant number from scratch just prior to the peak. Various measures are used to assess whether or not public safety requirements have been breached, and if so, then controls begin to be introduced. These controls are first on the street system and consist of street closures and movable barriers which constrain movement and densities. These are developed manually and this is the point at which those stakeholders who know the problem need to be involved with the model. Once we make such changes, however, the shortest routes and related accessibility surfaces also change and these must be recomputed so the entire sequence based on these two stages needs to be reiterated. Its essential logic is illustrated in Figure 2. This process continues until a steady state results and at this point, the model is essentially calibrated. Along the way, we may have ascertained that safety levels are too tight and that looser ones are possible or that safety measures are too slack and new ones required. In the latter, we can then initiate changes to the location of the parade and 
sound systems, thus beginning to use the model as a traditional means of testing alternative configurations of the Carnival.

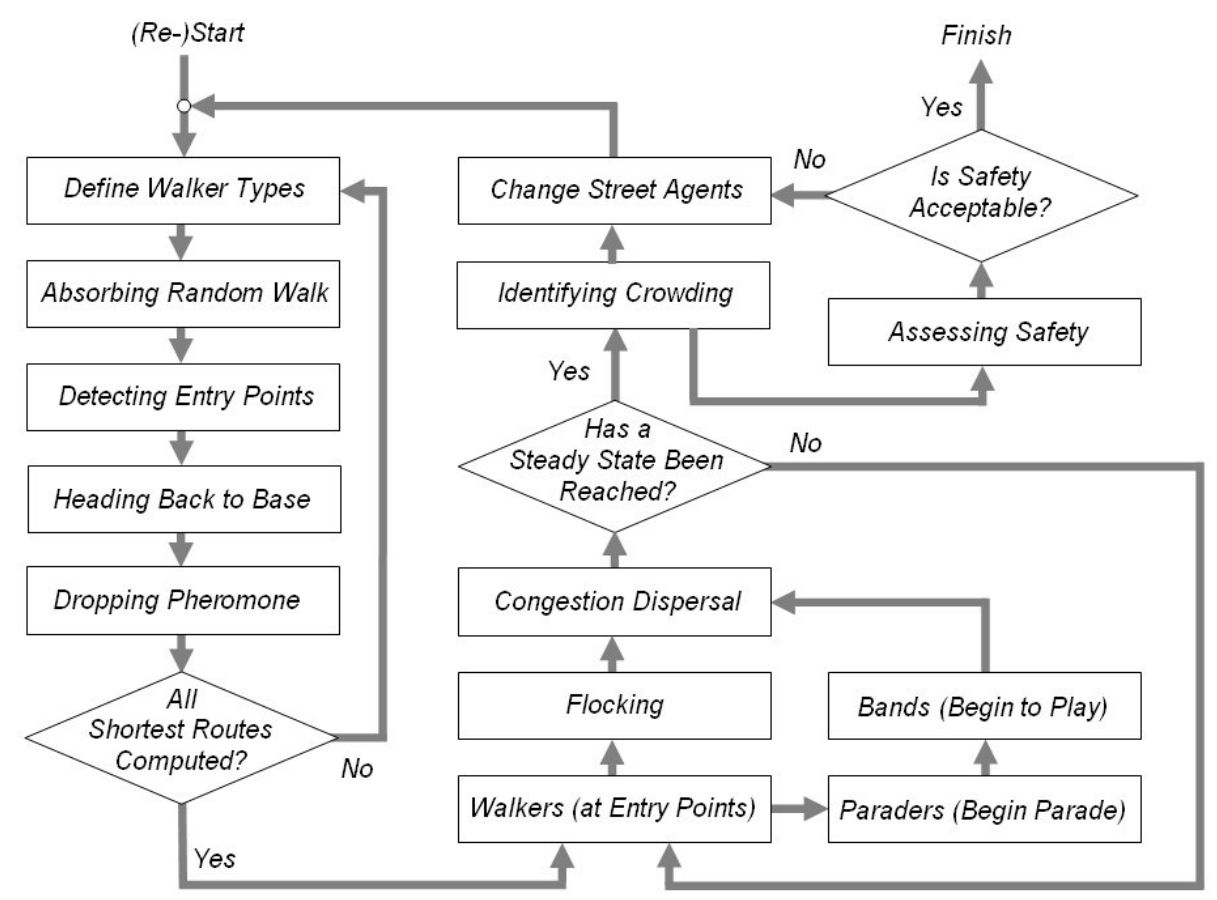

Figure 2: An Outline of the Two Stage Model

The first stage which is the 'swarm' algorithm is the initial left hand sequence, while the second stage simulation of crowding is the right hand sequence. Note how controls ('Change Street Agents') are gradually introduced through reiterating both stages of the entire sequence.

\section{The Mathematics of the Simulation}

All actions and interactions in the model take place in an event space composed of $N$ square cells indexed $i, j=1,2, \ldots, N$. The agent groups - walkers $(W)$, paraders $(P)$, bands $(B)$, and street objects $(S)$ are always located with respect to these cells at time $t=1,2, \ldots, T$ with $W_{i t}$ the number of walkers, $P_{i t}$ the number of paraders, $B_{i t}$ the number of bands, and $S_{i t}$ the number of street objects, in each cell $i$ at time $t$. In the case of bands and street objects, these are fixed in space and time and only a single 
agent is associated with each cell. For bands, $B_{i t}=1$ or 0 , that is a band exists in a cell or does not, and the total number of bands is thus $B=\sum_{i} B_{i t}, \forall t$. For streets, if $S_{i t}=1$, this means that the street agent is signaling that a cell is available for a walker to move to, that there are no obstacles to movement; if $S_{i t}=0$, obstacles such as barriers, buildings or street closures exist, and the cell is off-limits to movement. The total number of cells available is $S=\sum_{i} S_{i t}, \forall t$, and thus there are $\bar{N}=N-S$ cells which act as barriers or obstacles. The bands and street objects are entirely passive and do not change within a single run of the model. Street objects are however changed when control is being manipulated to meet safety measures through the outer loop in Figure 2 while band (and parade locations) are changed when different routing scenarios are evaluated.

The three types of walker are composed of those whose motive is to visit the parade $\left(W^{1}\right)$, those who primarily visit the bands $\left(W^{2}\right)$, and those who visit both $\left(W^{3}\right)$ with the total numbers of walkers in the system $W=W^{1}+W^{2}+W^{3}=\sum_{z=1}^{3} W^{z}$. Each individual walker is defined by the binary variable $W_{i t}^{k_{z}}$ which is set to 1 if a walker of type $z$ is in cell $i$ at time $t$, or 0 otherwise. A series of accounting relations define densities in cells and totals in the system at any one time, and the total number of walkers in each group $W^{z}$ is fixed over all time periods, implying that the overall total is also constant. Then the total number of walkers of type $z$ in $i$ are $W_{i t}^{z}=\sum_{k_{z}} W_{i t}^{k_{z}}$, the total in cell $i W_{i t}=\sum_{z} W_{i t}^{z}=\sum_{z} \sum_{k_{z}} W_{i t}^{k_{z}}$, and the total of type $z$ in the system $W^{z}=\sum_{i} \sum_{z} \sum_{k_{z}} W_{i t}^{k_{z}}, \forall t$. The same kinds of relations pertain to paraders except that there is only one type defined as $P_{i t}^{k}, k=1,2, \ldots, P$ which is equal to 1 or 0 depending upon whether or not a parader occupies cell $i$ at time $t$. The total paraders in cell $i$ is $P_{i t}=\sum_{k} P_{i t}^{k}$ and the overall total $P_{t}=\sum_{k} \sum_{k} P_{i t}^{k}=P, \forall t$.

The first stage of the simulation is based on the swarm algorithm which is solely based on walkers and does not involve paraders and bands except insofar as these act as sources for the random exploration of the street system. The walkers are launched 
from these sources $D$ (which are their ultimate destinations) at time $t=1$ where $W_{D 1}=\sum_{z} W_{D 1}^{z}=\sum_{z} \sum_{k_{z}} W_{D 1}^{k_{z}}$. Walkers of each type $z$ can move from cell $i$ to an adjacent cell $j$ in each time period $[t \rightarrow t+1]$ where $j \in \Omega_{i}$ and $\Omega_{i}$ is the eight cell neighborhood around $i$. In general, some of these cells will not be accessible because of obstacles but if the street agent signals an empty cell, that is $S_{j t}=1$, the agent is able to make a move. Note we now call the street agent $S_{j}$, dropping $t$ which does not change during the two stage simulation, and only changes on a longer cycle so that safety limits can be met. Movement from $i$ to $j$ in search of an origin $O$ is then determined by the relevant probability for type $z$ as

$$
p_{i j t+1}^{z}=\frac{\tau_{j t}^{z} S_{j}}{\sum_{\ell \in \Omega_{i}} \tau_{\ell t}^{z} S_{\ell}}
$$

$\tau_{j t}^{z}$ is the route accessibility to origins for agent type $z$, and this depends on the destinations which act as the sources of each walker type. A move from $i$ to $j$ is determined randomly according to the schedule of probabilities in (1). We also compute a composite accessibility surface for all the walkers $\eta_{j t+1}$ which in principle could be walker type specific but in practice, is more useful as an overall index combining the influences of all walkers. This is in contrast to $\tau_{j t}^{z}$ which, as we will see, is more like a density of movement associated with the use of routes. Then if $W_{i t}^{k_{z}}=1$ and $W_{j t+1}^{k_{z}}=1$, the accessibility surface to destinations is updated as

$$
\eta_{j t+1}=\eta_{j t}+\sum_{z} \sum_{k_{z}}\left(d_{D j t+1}^{k_{z}}\right)^{-\beta}
$$

where $d_{D j t+1}^{k_{z}}$ is the distance of walker $k_{z}$ from $D$ to cell $j$, and $\beta$ is a tunable parameter reflecting the friction of distance. In fact, this is also a 'sound surface' as the distance decay through the power law implied in (2), can be considered as a proxy for the decay of sounds from the attractor destinations. As we have implied earlier, in 
small spaces walkers are attracted to sounds and this is one way in which this feature can enter the model, at least implicitly. The analogy should not be taken too far as we have not attempted any analysis of sound decay with distance but the reason for using this surface is to incorporate system-wide effects which affect all walkers. Sound is one such effect. Finally at this point, the density of walkers at $j$ can be computed as $W_{j t+1}=\sum_{z} \sum_{k_{z}} W_{j t+1}^{k_{z}}$, or in terms of walker types, as $W_{j t+1}^{z}=\sum_{k_{z}} W_{j t+1}^{k_{z}}$.

The process implied by (1) and (2) continues until a walker discovers an origin $O$. For each walker $W_{j t+1}^{k_{z}}$, if $j \in \Omega_{O}$, the walker switches from exploratory to discovery mode $\bar{W}_{j t+1}^{k_{z}}$ and returns to the destination $D$ with knowledge of the discovery. The probability of returning is thus

$$
q_{i j t+1}^{k_{z}}=\frac{\pi_{j t}^{k_{z}} S_{j}}{\sum_{\ell \in \Omega_{i}} \pi_{\ell t}^{k_{z}} S_{\ell}}
$$

where $\pi_{j t}^{k_{z}}$ is based on the difference between the heading in the direction from $i$ to $j, \theta_{i j}^{k_{z}}$, and that from $i$ to the position defined by $W_{D 1}^{k_{z}}, \theta_{i D}^{k_{z}}$, which are combined as $\left[1+\left|\theta_{i j}^{k_{z}}-\theta_{i D}^{k_{z}}\right|\right]^{-1}$. This move is also chosen randomly and when $\bar{W}_{i t}^{k_{z}}$ moves to $\bar{W}_{j t+1}^{k_{z}}$, the walker marks the move by updating $\tau_{j t}^{z}$ as

$$
\tau_{j t+1}^{z}=\tau_{j t}^{z}+\sum_{k_{z}} \bar{W}_{j t+1}^{k_{z}}
$$

This process is akin to the walker dropping a marker or laying a pheromone trail when a discovery has been made: $\tau_{j t+1}^{z}$ measures the density which ultimately reflects a hierarchy, the highest of which form the shortest routes. When the walker comes within the neighborhood of its destination $j \in \Omega_{D}$, it switches back to exploration mode and the search begins over again. 
It takes some time before agents discover an origin. Before this, the search is a random walk with the route accessibility surface set as a uniform distribution, that is $\tau_{j t}^{z}=1$ until a time $t$ is reached when the first entry point is found. If a walker crosses the edge of the event space, it is absorbed, regenerates at its source destination, and begins its search again. In its early stages, this is a random walk with absorbing barriers with a standardized variance of distance traveled proportional to $t^{0.4}$, a little less than the value for an unconstrained random walk where $\sigma=t^{1 / 2}$ (Sornette, 2000). As the process continues, more and more origins are discovered while during exploration, walkers 'learn' to direct their search at routes to origins already discovered. Those origins closest to destinations are discovered first and the hierarchy of 'shortest routes' is thus built up, continually reinforced by this positive feedback. This is the process implied by the first inner loop in Figure 2. The algorithm is a variant of that observed in trail formation and collective foraging behavior amongst animal populations such as ants (Helbing, Schweitzer, Keltsch, and Molnar, 1997; Camazine et al., 2001). The swarms created are extremely efficient in predicting shortest routes in geometrically constrained systems (Bonabeau, Dorigo, and Theraulaz, 1999). Here we do not let the pheromone trail $\tau_{j t}^{z}$ decay, while the accessibility surface $\eta_{j t}$ gives the relative attraction of destinations to different street locations in terms of distance and its proxy as noise. The exploratory stage finishes when the differences in path densities $\tau^{z}=\sum_{j}\left|\tau_{j t+1}^{z}-\tau_{j t}^{z}\right|$ and $\tau=\sum_{z} \sum_{j}\left|\tau_{j t+1}^{z}-\tau_{j t}^{z}\right|$ fall below various predetermined thresholds which we fix through experimentation. This marks the convergence of the swarm algorithm and the end of the first stage at time $T$.

In the second stage, we launch the walkers from their entry points, and these walkers move towards the event using the surfaces $\tau_{j T}^{z}$ and $\eta_{j T}$ as indicators of accessibility. These are aggregates from the entire time sequence. We suppress $T$, normalize these as $\tau_{j}^{z}$ and $\eta_{j}$ and then combine them as $\left(\tau_{j}^{z}\right)^{\alpha} \eta_{j}^{1-\alpha}$. There are of course many ways in which we could do this and there is inevitably an element of double counting in their joint usage. We could even keep these as separate surfaces for each walker 
group. However we use this parameterization as being one of the most straightforward ways in which different types of accessibility can be merged, the best combination of which will reflect the best fit of the model to the observed situation. The basic probability of movement for each walker type $z$ is now defined as

$$
q_{i j t+1}^{k_{z}}=\frac{\left(\tau_{j}^{z}\right)^{\alpha} \eta_{j}^{1-\alpha} S_{j}}{\sum_{\ell \in \Omega_{i}}\left(\tau_{\ell}^{z}\right)^{\alpha} \eta_{\ell}^{1-\alpha} S_{\ell}}
$$

where $\alpha$ is a tunable parameter which plays a role similar to homogenous production functions of degree 1 such as the Cobb-Douglas, widely used in micro-economics for their scaling properties (Henderson and Quandt, 1980). We use (5) to select directions of movement from $i$ to $j$ where we use each probability $q_{i j t}^{k_{z}}$ in the neighborhood $\Omega_{i}$ to determine the direction $j$ in which the walker moves. This is done randomly with new headings in the direction $j$ computed as $\bar{\theta}_{i t+1}^{k_{z}}$ and then used to update the existing heading as $\hat{\theta}_{i t+1}^{k_{z}}=\lambda \bar{\theta}_{i t+1}^{k_{z}}+(1-\lambda) \theta_{i t}^{k_{z}}$ where $\lambda$ reflects a lag in response.

There are two effects that complicate this movement. The first is herding or flocking (Reynolds, 1987; Vicsek, Czirok, Ben-Jacob, Cohen, and Shochet, 1995). This directs movement as an average of all movement in the immediate neighborhood reflected in the headings where $\theta_{i t+1}^{k_{z}}=\sum_{z} \sum_{l_{z} \in j} \sum_{j \in \Omega_{i}} \hat{\theta}_{j t+1}^{l_{z}} W_{j t}^{l_{z}} / \sum_{z} \sum_{l_{z} \in j} \sum_{j \in \Omega_{i}} W_{j t}^{l_{z}}$. However a move by walker $W_{i t}^{k_{z}}$ to $W_{j t+1}^{k_{z}}$ only takes place if the density of walkers in cell $j$ is less than some threshold $\Psi \leq \sum_{z} \sum_{k_{z}} W_{j t}^{k_{z}}=2$ based on the accepted standard of 2 persons per meter squared $\left(\mathrm{ppm}^{2}\right)$ (Fruin, 1971; Still, 2001). If this is exceeded, the walker evaluates the next best direction and if no movement is possible, remains stationary until the algorithm frees up space on subsequent iterations. These rules are ordered to ensure reasonable walking behavior. There are many variants that can be tried but those adopted seem to be plausible from ad hoc observation and from the literature. This, however, represents a major area for further research in pedestrian modeling in particular and agent modeling in general. 
The paraders $P_{i t}^{k}$ move in a much more structured manner, around a parade loop defined by a linear sequence of cells $\{i\}$ forming the set $i \in \Pi$ which are ordered so that there is only one direction of movement from $[i \rightarrow j]$ in time $[t \rightarrow t+1]$. Headings and probabilities of movement do not have to be calculated for the paraders although movement is determined with some random input. Although the floats normally travel to adjacent cells in the given direction with the flow controlled to give reasonable moving behavior in time, this smoothness does incorporate a degree of intermittency. At any time $t$, the total number of paraders in each cell $i$, $P_{i t}=\sum_{k} P_{i t}^{k}, i \in \Pi$, are distributed approximately uniformly amongst the total number of cells used for the parade $\left(\sum_{i \in \Pi} 1\right)$. A parader will progress to an adjacent cell in a time period according to a random function which ensures that most paraders make such transitions but a few do not and stay in the cell that they are currently in. For any parader $P_{i t}^{k}$, then if $\operatorname{rand}(1)<\Theta$, then $P_{j t+1}^{k}=P_{i t}^{k}$ otherwise $P_{i t+1}^{k}=P_{i t}^{k}$. If the threshold $\Theta$ is set a little less than 1 , then most paraders will move smoothly to the next cell. The parameter is set experimentally to introduce a level of intermittency in the flow of the parade that is observed in practice and which, for the most part, avoids major incidents.

There are of course potential conflicts between paraders and walkers when they come into contact in the neighborhood which is immediately adjacent to each cell occupied by the parade. We define cells in these neighborhoods as $j \in \Phi_{i}^{P}$ where we compute the density of walkers in these cells. Note that walkers cannot occupy cells which define the parade route. Then if $\sum_{j \in \Phi_{i}^{P}} \sum_{z} \sum_{k_{z}} W_{j t}^{k_{z}} \geq \Lambda$, where $\Lambda$ is the critical density in cells adjacent to the parade, then walkers disperse in the same way that they do when they interact with each other and breach critical density limits. In fact we keep a trace $A_{i}^{P}(t)=\sum_{t^{\prime}=1}^{t} \sum_{j \in \Phi_{i}^{P}} \sum_{z} \sum_{k_{z}} W_{j t^{\prime}}^{k_{z}}$ which provides us with a record of potential accident hot spots along the parade. The same kind of logic is used in relation to crowding around the fixed sound systems where the bands are playing. In analogous fashion, we define cells in neighborhoods around each band $i$ as $j \in \Phi_{i}^{B}$, 
an equivalent test for dispersion as $\sum_{j \in \Phi_{i}^{B}} \sum_{z} \sum_{k_{z}} W_{j t}^{k_{z}} \geq \Xi$, and the trace of potential accidents as $A_{i}^{B}(t)=\sum_{t^{\prime}=1}^{t} \sum_{j \in \Phi_{i}^{B}} \sum_{z} \sum_{k_{z}} W_{j t^{\prime}}^{k_{z}}$. This second stage is terminated when the density of walkers enters a steady state which means that the walkers are moving in the area of the Carnival and that movement between attractions is beginning to repeat itself. We can test for this using various criteria such as the statistics which we present below. A generic test however is based on the lagged density difference $\xi_{z}^{T-t^{\prime}}=\left\{\sum_{i} \sum_{t=1}^{t^{\prime}} \sum_{k_{z}}\left[W_{i T-t}^{k_{z}}-W_{i T-t-1}^{k_{z}}\right]\right\} /\left\{T-t^{\prime}-1\right\}$ defined for each type of walker $z$ (or for all walkers) where the summation over time is from the point where the simulation enters the steady state $t^{\prime}$ to the point where the simulation ends $T$. This formula handles the case where periodicity is feature of the simulation.

We can now assess how good the model is at predicting the observed distribution of crowds. We do not define any statistics for the individual groups $z$ for two reasons. First, we do not have good data on these differences with respect to origins and destinations for we take this distribution from the observed locations around the various attractions over the Carnival period. As even those visitors whose prime concern is to visit either bands or the parade but not both, are likely to visit each of these, then our observations do not directly tie in with motivations. Thus we will only work with aggregate quantities. We compare the predicted density of all walker types $W_{i t}$ and average neighborhood density $\widetilde{W}_{i t}=\sum_{z} \sum_{k_{z} \in j} \sum_{j \in \Omega_{i}} W_{j t}^{k} / \sum_{j \in \Omega_{i}} S_{j}$ in cells where observed densities are available. We then relate these to the number of occupied cells $\sum_{i} n_{i}=M$ (where $n_{i}=1$ if $W_{i t}^{k_{z}}>0$, otherwise $n_{i}=0$ ) and the number of available cells $\sum_{i} S_{i}=S$, defining averages as $\rho(t)=\sum_{i} \widetilde{W}_{i t} / \sum_{i} n_{i}$, $\sigma(t)=\sum_{i} \widetilde{W}_{i t} / \sum_{i} n_{i}$ and $\vartheta(t)=\sum_{i} n_{i} / \sum_{i} S_{i}$. For different threshold values $\Psi$, if $W_{i t}>\Psi$, then $c_{i t}(\Psi)=W_{i t}$ otherwise $c_{i t}(\Psi)=0$, and the proportion of the population at risk is thus $Z_{t}(\Psi)=\sum_{i} c_{i t}(\Psi) / M$. Average distance traveled in each time period $[t \rightarrow t+1] \quad$ is $\quad U_{t+1}=\sum_{z} \sum_{i j k_{z}} d_{i j t+1}^{k_{z}} / M \quad$ with the percent actually moving $V_{t+1}=\sum_{z} \sum_{i j k} W_{i t}^{k_{z}} W_{j t+1}^{k_{z}} / M$. 
These two stages define the complete model with subsequent stages being generated through reiteration of the entire sequence. These additional stages are activated if the statistics generated at the end of the second stage suggest that public safety is compromised. It consists of examining the statistics from the second stage, and gradually making changes to reduce the population at risk by introducing barriers, capacitating entry points, and closing streets. This is achieved by changing the number of street objects - switching street agents off or on that signal how much space for movement is available. The set of agents $\left\{S_{j}\right\}$ is updated to $\left\{S_{j}^{\prime}\right\}$ where the prime indicates that this is the next iteration in the sequence with the model beginning again and the original time $t$ being indexed back at $t=1$. As the repercussions of these changes are not immediately obvious, we make these changes one by one forming $S_{j}^{\prime}, S_{j}^{\prime \prime}, S_{j}^{\prime \prime \prime}, \ldots$, rerunning the model each time until an acceptable solution emerges. These reiterations assume that the shortest routes surface needs to be updated and this involves rerunning the entire two stage procedure shown in Figure 2.

\section{Dimensioning the Problem and Calibrating the Model}

The main feature of the Carnival is the parade which involves 89 floats and 64 support vehicles that continuously move around a closed loop of $4.9 \mathrm{kms}$ from noon until dusk on each of the two days of the event. Within the loop, there are 42 static sound systems - bands - and across the entire area, some 240 licensed street stalls with wellresourced health and comfort points for visitors and paraders alike. Most visitors to the event use public transport to reach the 38 entry points which define the zone within which traffic is excluded by the police, some $40 \%$ using the tube and $22 \%$ using buses. Many others walk from central London or neighboring areas and only a small number $(<10 \%)$ travel to the Carnival by car or taxi. The street system, and the entry, parade, static sound, and tube (subway) station locations, are shown in Figures $3 \mathrm{a}$ and $3 \mathrm{~b}$ respectively. 


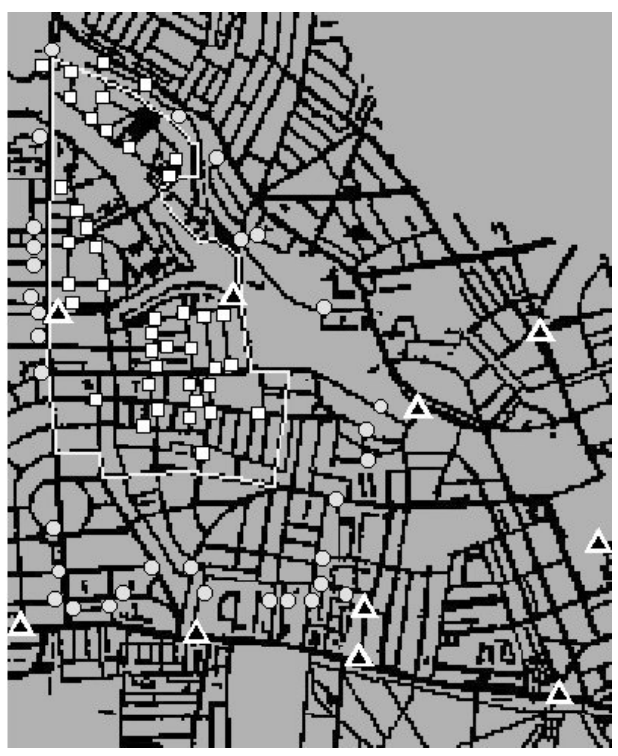

a The Street Geometry in Notting Hill

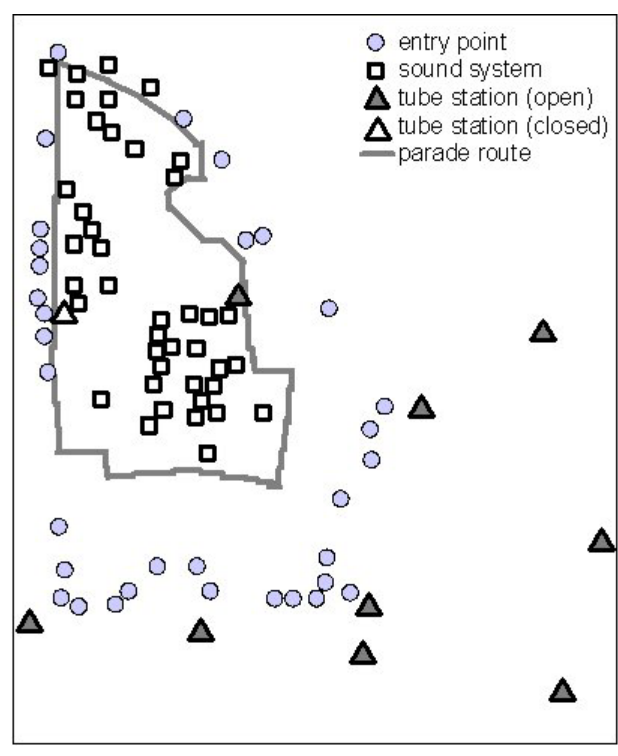

b Entry, Tubes, Parade, and Bands

Figure 3: Geometric and Locational Features of the Carnival

The number of visitors grew dramatically in the 1990s reaching 1.2 million (over the two day period) in 1999 but dropping to something like 700,000 last year (2001). Informed speculation suggests that this is possibly due to the negative publicity associated with high levels of crime at the event which have dominated policing in recent years. Problems of crowding have become significant with accidents due to congestion rising dramatically and problems of emergency vehicle access being compounded by the conflict between the parade and visitors entering the inner core of the area to visit the sound systems. In 2001, there were 500 accidents (100 requiring hospital treatment with 30 percent related to wounding) and 430 crimes with 130 arrests. Three fatalities occurred in 1999 from violent crime. This and the need to review resources allocated to manage the event - some 3500 police and stewards were required each day - was the trigger that led to the review that initiated this technical work (CRG, 2001).

Attendance is more than twice as large on the second day with the peak level between $4 \mathrm{pm}$ and $6 \mathrm{pm}$ when around 260,000 visitors are in the Carnival area. During the event, there is considerable movement between the various attractions and although 
precise movements are not known, the rate of 'churn' which is ratio of those entering and leaving the area to those within, is around 40 percent. In terms of the volume of movement from entry points to attractions, four streets located at E-W-N-S of the area account for over 50 percent. Crowd densities are high at about 0.25 persons per $\mathrm{m}^{2}$ of which $0.47 \mathrm{ppm}^{2}$ line the parade route and $0.83 \mathrm{ppm}^{2}$ lie inside. Critical densities up to $1 \mathrm{ppm}^{2}$ exist in the central section where many of the bands are clustered while around the judging point in the south west area of the parade, route densities rise to $1.3 \mathrm{ppm}^{2}$ which are critical.

The data we have available was collected at the Carnival in 2001 specifically for this analysis but was limited by resources and technology. Essentially origin and destination data is available, the origins from a cordon count at all 38 entry points throughout the 2 day period, while destination data along the parade and at some static sound locations was derived from video footage taken from police helicopters during the late afternoon of the second day (IPS, 2002). From this footage, 1022 images have been extracted from which densities have been manually computed and averaged to cells as indicated in Figure 4. Additional data on entry and exit volumes at the subway station from surveys by London Underground Ltd., and bus volumes at setting down locations have been integrated into the density database. What we do not have are the paths that walkers take from entry points to attractions and the movements between the various attractions when visitors are within the Carnival area. Automatic ways of collecting such data are practically impossible for laser scanning is too expensive and CCTV is legally forbidden for this kind of monitoring. These constraints led us directly to the first stage of the simulation which is concerned with reconstructing such paths.

To set up the simulation using this data, we need to decide on the level of resolution of the space within which the Carnival is to be modeled. This is largely dictated by software considerations in that the software used has upper limits on the number of agents and cells that can be handled. No more than 16,000 agents can be simulated on no more than 52,500 cells. Thus the area of the space was set as 207 x 251 pixels and 13,000 agents were defined, divided into those whose intention is to visit the bands (3000), those visiting the parade (5000), and those visiting both (5000). This must be 
interpreted as a 5 percent sample of the 260,000 visitors in the peak period that is being modeled and thus all quantities must be scaled and adjusted accordingly. This is less than satisfactory because we consider that a fully-fledged model should be able to handle not a sample but the full population of agents. This however would require considerable programming that was simply not possible within this project at this time but it remains is a longer term aim of this research. The cell structure is implied by the pixelation shown in Figure 3. Key locational features of the problem - entry points and Carnival destinations (parade and bands) shown in Figure $3 \mathrm{~b}$ - represent the sources for the swarm algorithm which generates the paths in the first stage of the model and thus the starting (entry) points for the visitors in the second stage.
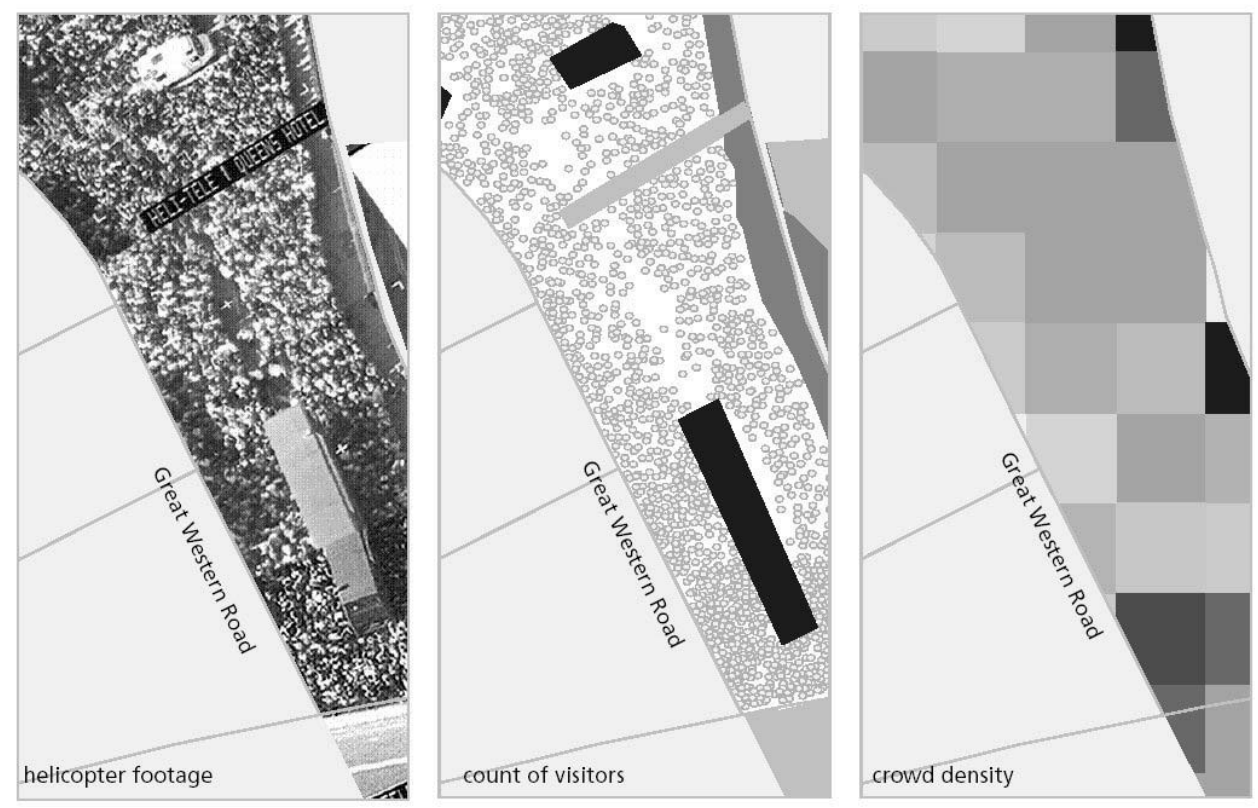

Figure 4: Translating Video Footage of Walkers to Cell Densities

Like all calibrations, the purpose is to define values for the parameters of the model (whose values are unknown at the outset of the simulation) so that the predictions of the model are as close as possible to the observed data. In terms of the model in equations (1) to (5), we have fixed several parameters relating to lags and crowding at what we consider reasonable values. This enables us to reduce the search problem to a phase space defined by only two parameters - the friction of distance $\beta$ and the accessibility weighting parameter $\alpha$. The density thresholds for walkers with respect 
to each other $\Psi$, to paraders $\Lambda$, and to bands $\Xi$ have all been set at $2 \mathrm{ppm}^{2}$ as suggested in official advice (Fruin, 1971; ISP, 2002). In the second stage of the simulation once paths and accessibilities have been defined, the lag in updating the heading from the previous value is set at $\lambda=0.4$ while the intermittency threshold for moving the elements in the parade forward is set at $\Theta=0.9$. The calibration thus consists in running the model through its two stages (together with any subsequent control) over samples of parameter values $\alpha, \beta$ until a combination is found which optimizes the model's predictions based on the fit of observed to predicted densities.

The simulation starts with the three types of walker $W_{D 1}^{z}$ located at the Carnival event locations $D$, the ultimate destinations of the walkers. They begin their random walk through the streets in search of entry points where they will actually start their walk to the Carnival in the second stage. To illustrate the power of the swarm algorithm around which this walk is structured, we show the kinds of paths that are generated for walkers $(z=2)$ starting from the sound systems $\left(W_{D 1}^{2}\right)$ and finding the entry points but in the absence of any geometric constraints posed by the streets. In short, we set the streets $S_{j}=1, \forall_{j}$, and in this way the walkers find the entry points directly. They move out in concentric rings from the static sound locations, the symmetry of this concentricity being broken when entry points are first discovered. In Figures 5a and $5 \mathrm{~b}$, we show the location of the walkers at times $t=10$ and $t=50$. By $t=500$, the pattern is dominated by the paths between sound systems and entry points as shown in Figure 5c, and this is confirmed by the straight line traces based on the densities $\tau_{j T}^{2}$ reproduced in Figure $5 \mathrm{~d}$. All the results in this section are based on simulations with the best combination of $\alpha, \beta$ where $\beta=0.65$, and $\alpha=0.35$. With the streets in place, the three shortest route hierarchies $\tau_{j T}^{z}, z=1,2,3$ are shown in Figures $6 \mathrm{a}$ to $6 \mathrm{c}$ together with the overall accessibility surface $\eta_{j T}$ in Figure $6 \mathrm{~d}$ computed from equation (2). These show the hierarchy of routes which are then combined into a composite accessibility surface used to initiate the actual walks to the Carnival which is the basis of the second stage of the model. 


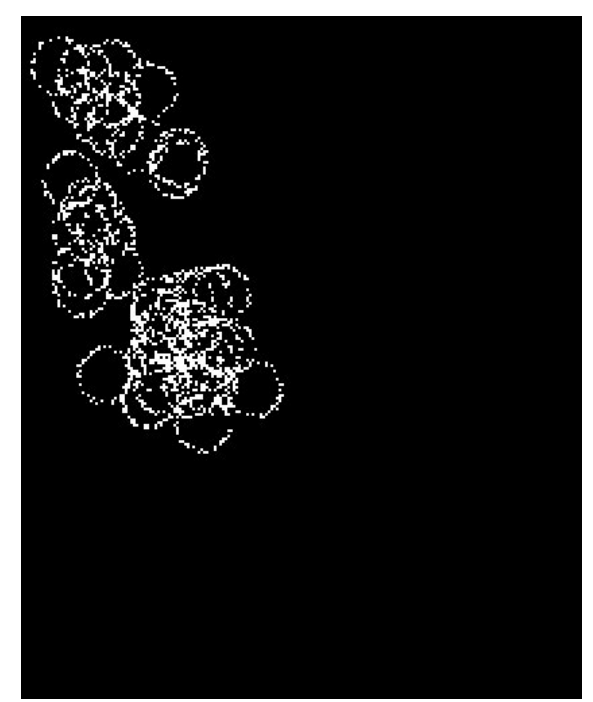

a Swarm at $t=10$

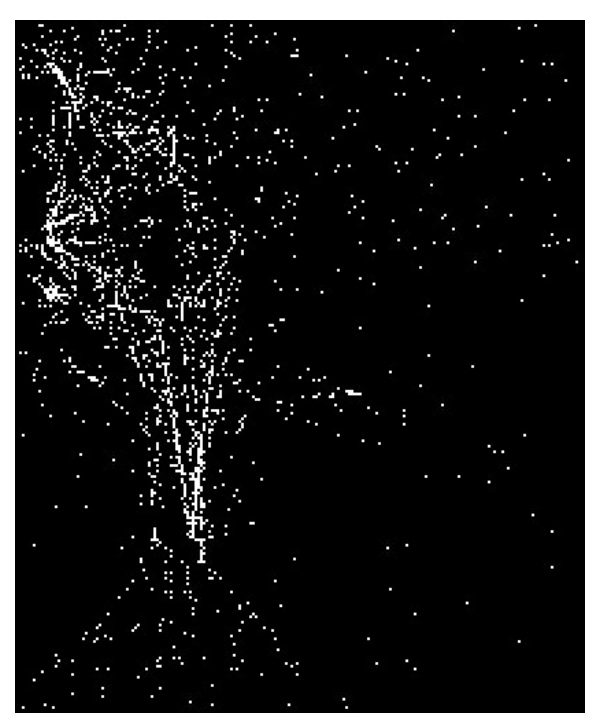

$c$ Swarm at $t=500$

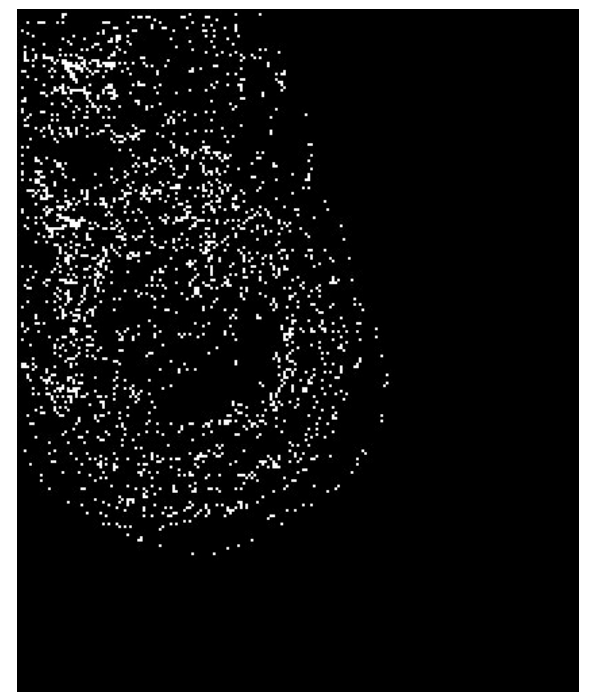

$b$ Swarm at $t=50$

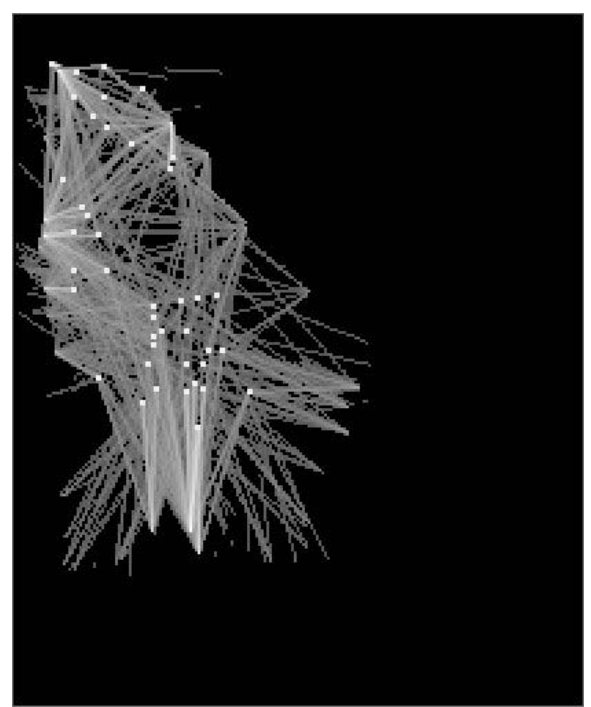

$d$ Density of Shortest Paths

Figure 5: Swarming in Search of Entry Points without any Street Geometry

We will examine the various statistics associated with these simulations in more detail below after we have examined the second and subsequent stages of the simulation where safety levels determine how control is developed as part of the modeling process. Using the accessibility surface shown in Figure 6d, we locate all walkers at the points where they are observed to enter the Carnival area (at the entry points in Figure $3 \mathrm{~b}$ ) and we then launch these using the access surface to guide the directions that they walk. Flocking and congestion dispersal are an integral part of this second 
stage simulation as are the interactions between walkers and paraders and walkers and the bands. We have not included emergency vehicles as separate agents for we consider the scale of the simulation and the fact that we are running this at a relatively crude level of cell resolution tends to make such interactions between walkers, paraders, bands, and emergency vehicles somewhat arbitrary. In fact, a clear problem in the Carnival is the fact that accidents which are caused by congestion can be further exacerbated by the difficulties of getting emergency vehicles to attend to injured walkers and paraders but explicit consideration of these effects must await the

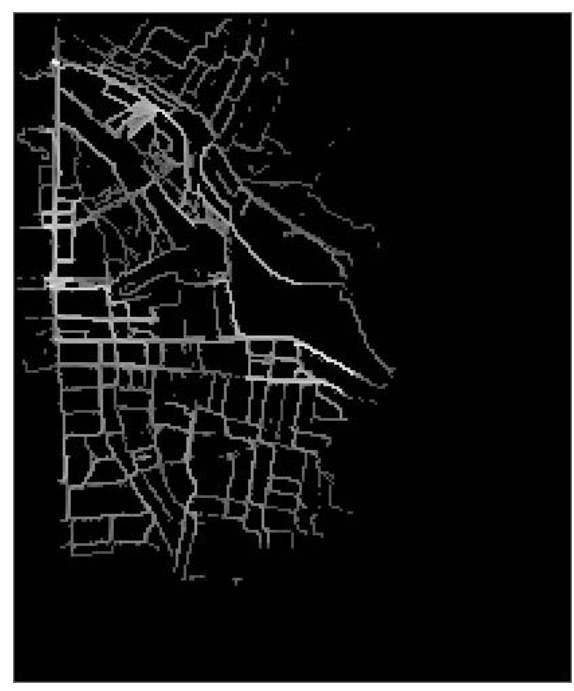

a $z=1$ Agents Visiting Bands

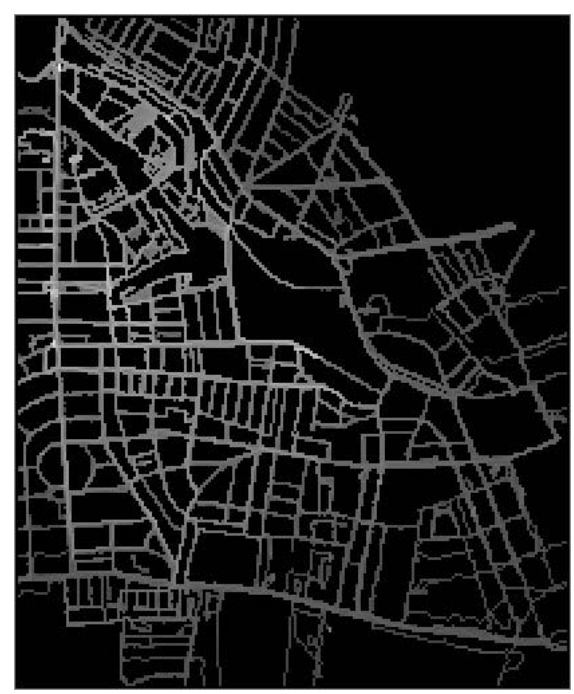

c $z=3$ Agents Visiting Bands/Parade

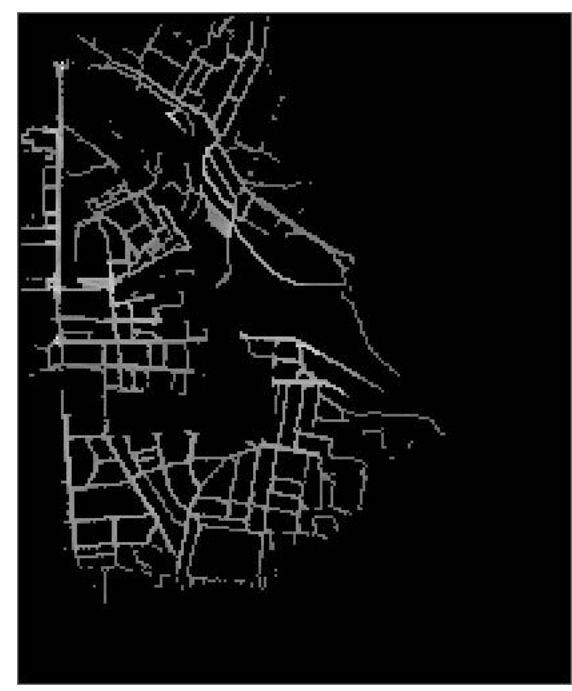

$b \quad z=2$ Agents Visiting Parade

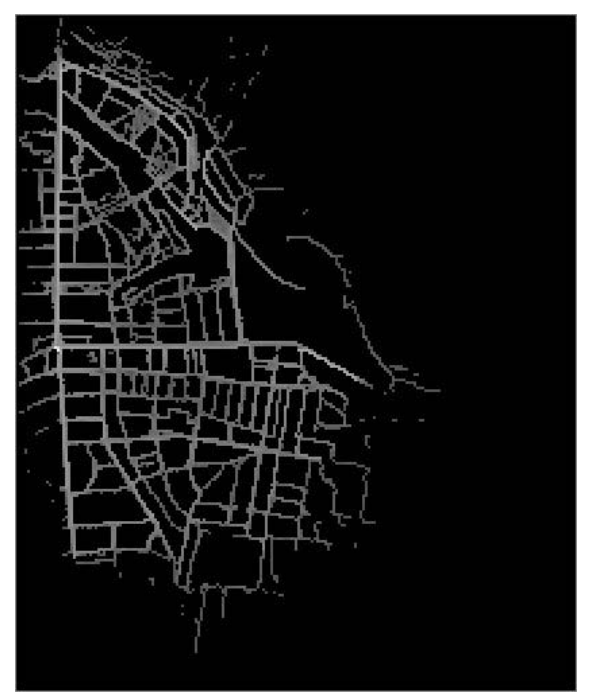

d Composite Accessibility Surface

Figure 6: Hierarchies of Shortest Routes and Associated Accessibility 
detailed model that we are currently constructing with more appropriate software. In Figure $7 \mathrm{a}$, we show the composite access surface computed for just one of the walker types - those who visit the parade and the bands $(z=3)-$ as $\left(\tau_{i}^{3}\right)^{0.35} \eta_{i}^{0.65}$ where we use $\alpha=0.35$, the best values determined through running the entire model through different combinations of its parameter values within its phase space. We show the movement of all three walker types at $t=100$ and then in the steady state at $t=500$ in Figure $7 \mathrm{~b}$ and $7 \mathrm{c}$.

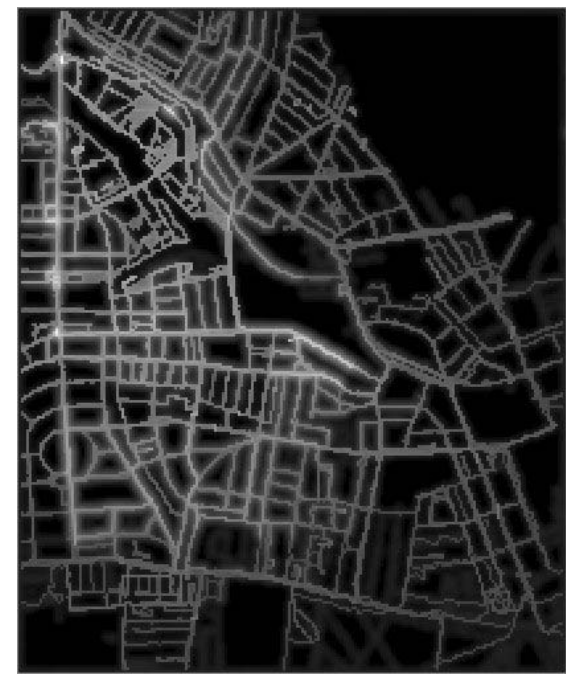

a Composite Access Surface $z=3$

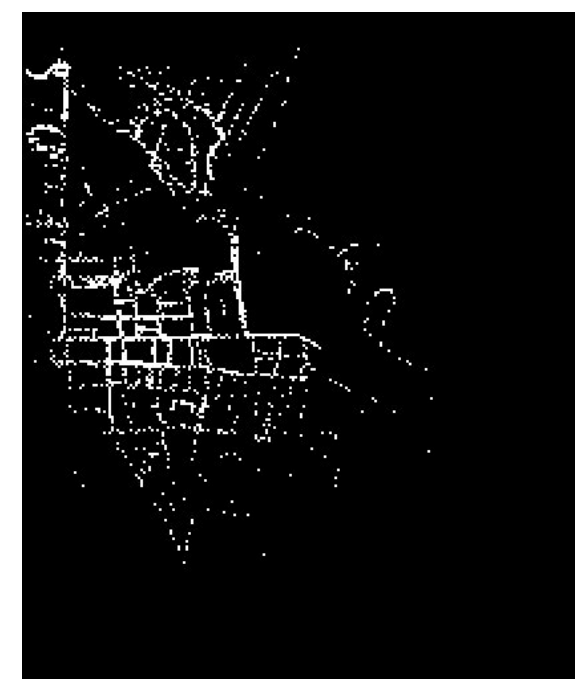

c Near the Steady State Distribution

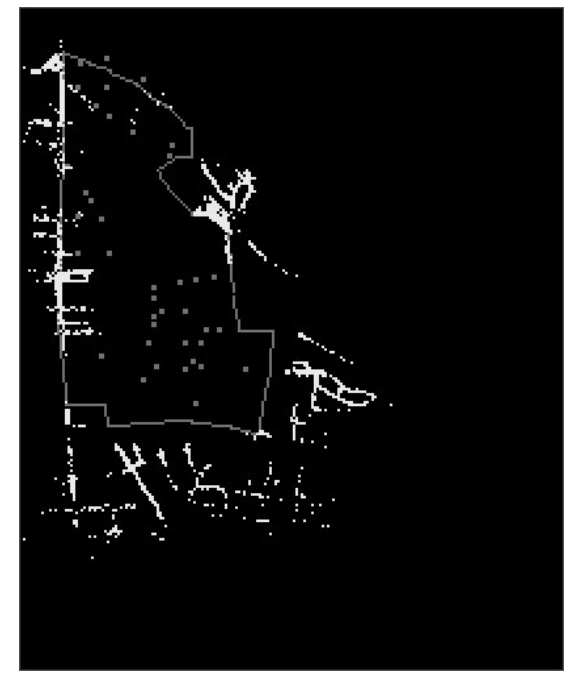

$b$ Agents Leaving the Entry Points

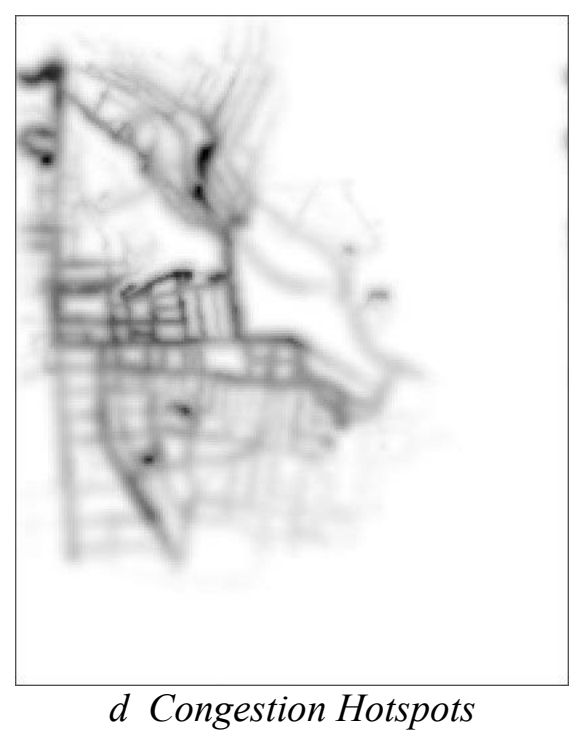

Figure 7: The Second Stage of the Simulation 
In the steady state, we have also computed the hotspot trace indices $A_{i}^{P}(t), A_{i}^{B}(t)$ defined earlier as well as those for walkers not in the parade or band locations $A_{i}^{W}(t)=\sum_{t^{\prime}=1}^{t} \sum_{j \in \Omega_{i}} \sum_{z} \sum_{k_{z}} W_{j t^{\prime}}^{k_{z}}$. We have added these surfaces and smoothed them through diffusion as shown in Figure $7 \mathrm{~d}$ which gives a good indication of the accident and crowding hotspots associated with the simulation at the end of its second stage. It is this kind of picture that is essential in determining where safety controls are to be introduced. In fact the simulation reveals that a mismatch between actual hot spots and those produced by the simulation. Congestion along the parade itself in the southwest corner is not as problematic as actually observed in 2001 while there are more serious problems in the northern part of the route. The model does tend to give greater weight to the northern area and this is a systematic error that needs to be addressed in new versions of the simulation.

At the end of the second stage, we assess safety levels. To illustrate how we proceed, we have introduced the various barriers that are associated with the actual Carnival in 2001. The core area in the center of the parade route is reserved as an area for police and emergency management in case a serious disaster occurs and various other roads are closed for resident access and due to constrictions in streets unsuited to large crowds. The areas are shown in Figure 8a. All the previous kinds of visualizations are available for running the model with these barriers imposed but as these add little to the picture so far, we simply produce a summary of density levels which provide some idea how the crowding problems of the second stage are resolved. These are shown in smoothed surface in Figure $8 \mathrm{~b}$ where it is clear that the north east leg of the parade is problematic in terms of crowding as is the center area of the Carnival where many static sound systems are clustered. In fact, the existing crowd control through barriers and street closures does go some way to reduce the crowding problems although it is probably best to consider the map of hot spots in Figure $8 \mathrm{~b}$ as a diagnostic for future action rather than a definitive analysis of serious problem locations. A clearer analysis of safety must be focused on the actual densities which we will now examine using graphs of how density levels change through the various simulations. 


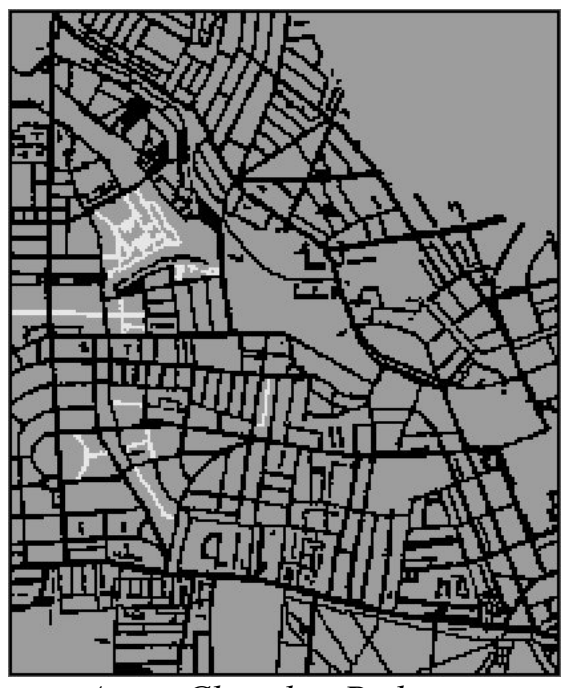

a Areas Closed to Pedestrians

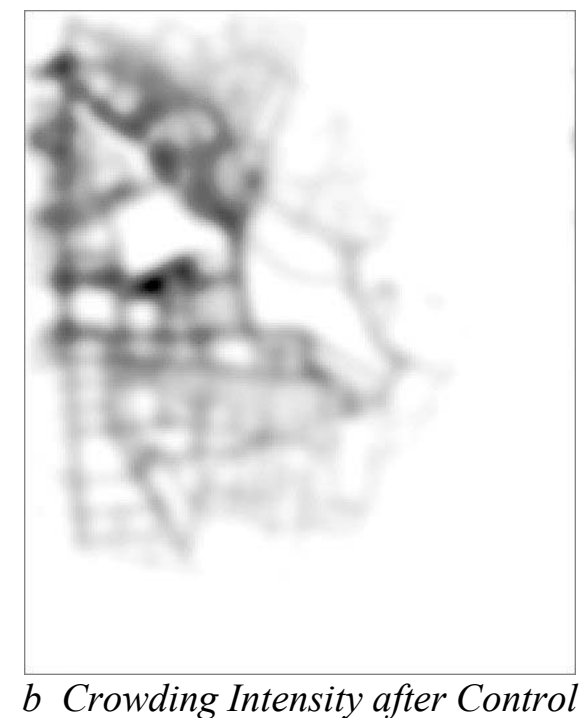

$b$ Crowding Intensity after Control

Figure 8: Control of Crowds After the Second Stage Simulation

\section{Safety, Policy and Scenario Testing}

Before we examine safety issues and provide some sense of how the model is being used in evaluating different routes for the parade which reduce congestion, the performance of the existing model must be examined. At each stage of the simulation, we can compare existing densities of walkers in their steady state with observed densities at 120 locations which we have extracted as being significant from the density database. In the first stage however, the swarm algorithm predicts the numbers of walkers who 'find' each entry point, and from the cordon survey (ISP, 2002), we are able to account for $64 \%$ of this variance; that is, of the numbers observed entering the Carnival area during the peak period from $4 \mathrm{pm}$ to $6 \mathrm{pm}$ on the second day, the swarm algorithm predicts that the number of agents who find these points is correlated at $64 \%$ with the observed totals. The second stage which involves locating the observed walkers at these entry points and then enabling them to move to the Carnival attractions leads to $72 \%$ of the variance of observed densities in the 120 locations being explained. These predictions not only relate to the sound systems but to selected points on the parade route for which density data is available. At the third stage, when the model is rerun with the official street closures and barriers imposed, the variance 
explained increases to $78 \%$, but not all the points of extreme crowding have been removed, as we have already shown in Figure $8 \mathrm{~b}$.

In terms of safety implications from these linked simulations, we graph the key indicators in Figure 9 for the entire system. These statistics are averages and totals and to understand their impact locally, reference to the densities and hotspots in Figures 6, 7 and 8 must be made. Nevertheless, besides showing how the simulation works, the trajectories in Figure 9 give some sense in which densities change. There is a critical distinction between the densities and occupancies associated with the first and second stages of the model - between the swarm and climbing algorithms. The swarm algorithm begins with all walkers at destination attractions and as these spread out in the search for entry points, densities drop dramatically as illustrated in the upper and lower left hand graphs in Figure 9. As the algorithm converges on the most favored shortest routes between entry and attraction points - origins and destinations, the percent of the walkers who breach the critical thresholds of 0.5 and $1 \mathrm{ppm}^{2}$, continuously increases. Although there is some sense of convergence to stable values, these percentages continue to rise as more and more walkers are attracted to the shortest routes. The percentage who occupy the streets is fairly stable as is the average distance traveled in each time period. In all cases, there is continual volatility in these trajectories as local geometric factors perturb the movement of walkers. Nowhere in models such as these is there a smooth flow of walkers, and this seems characteristic of what actually happens as walkers move between events at the Carnival itself.

In contrast, the second stage of the model displays quite different behavior. Walkers this time move from their entry points by climbing the accessibility surface towards the attractions. Again walkers begin at very high densities which fall off as they spread out from their origins. These densities then rise slowly, peaking as walkers enter the Carnival area and move between attractions. The graphs of average point and neighborhood densities and critical threshold values converge to quite stable values which mark the steady state as the graphs on the right hand side of Figure 9 show. What is of interest is that the average distance traveled slightly increases as walkers enter the steady state and the volatility of walkers gets greater as they crowd together around the Carnival attractions. A key issue of course is the difference 
between the simulation without any controls on street closures and that which develops when routes are closed as indicated in Figure 8a. The results are encouraging in that it is clear that density levels at each point and in each neighborhood are reduced on average by around 12 percent with the percent vulnerable to crowding at $0.5 \mathrm{ppm}^{2}$ and $1 \mathrm{ppm}^{2}$ reduced by about 14 percent. This must be attributed solely to the effect of closures and related controls. In short, this suggests that existing controls are effective although it also suggests that there is still considerable room for greater safety measures which would reduce the percent subject to such crowding to much lower levels. As Figure 9 shows, existing controls show that 20 percent breach the $Z_{t}(\Psi)>0.5$ and 8 percent the $Z_{t}(\Psi)>1$ thresholds.

For reasons of both space and confidentiality, we are not able to present the detailed results of using the model to assess the impact of alternative parade routes. However it is worth presenting some general comment on the way the model has been used. The process of developing six different alternative routes was based on a series of meetings organized by the Greater London Authority's Carnival Review Group (CRG, 2001) and from this process, six somewhat different routes emerged for testing. These routes essentially broke the circularity of the existing route. The simplest was based on an L-shaped procession along the existing north-south route (Ladbroke Grove) and then west-east along the Bayswater Road (at the bottom of the map in Figure 3a) finishing in Hyde Park. The other five routes were variations either on this or on the existing route. The key issue in running the model however is in determining visitor volumes at entry points with the location of the sound systems unchanged. In fact a series of related models which we will not describe here, were built to predict these volumes based on linear regression of observed volumes against key route factors such as visibility, accessibility, and various distance measures to related facilities (ISP, 2002). Thus for any location in the area, it is possible to predict visitor volumes which when normalized to total visitor numbers, give the numbers entering the Carnival area associated with any new parade route. 


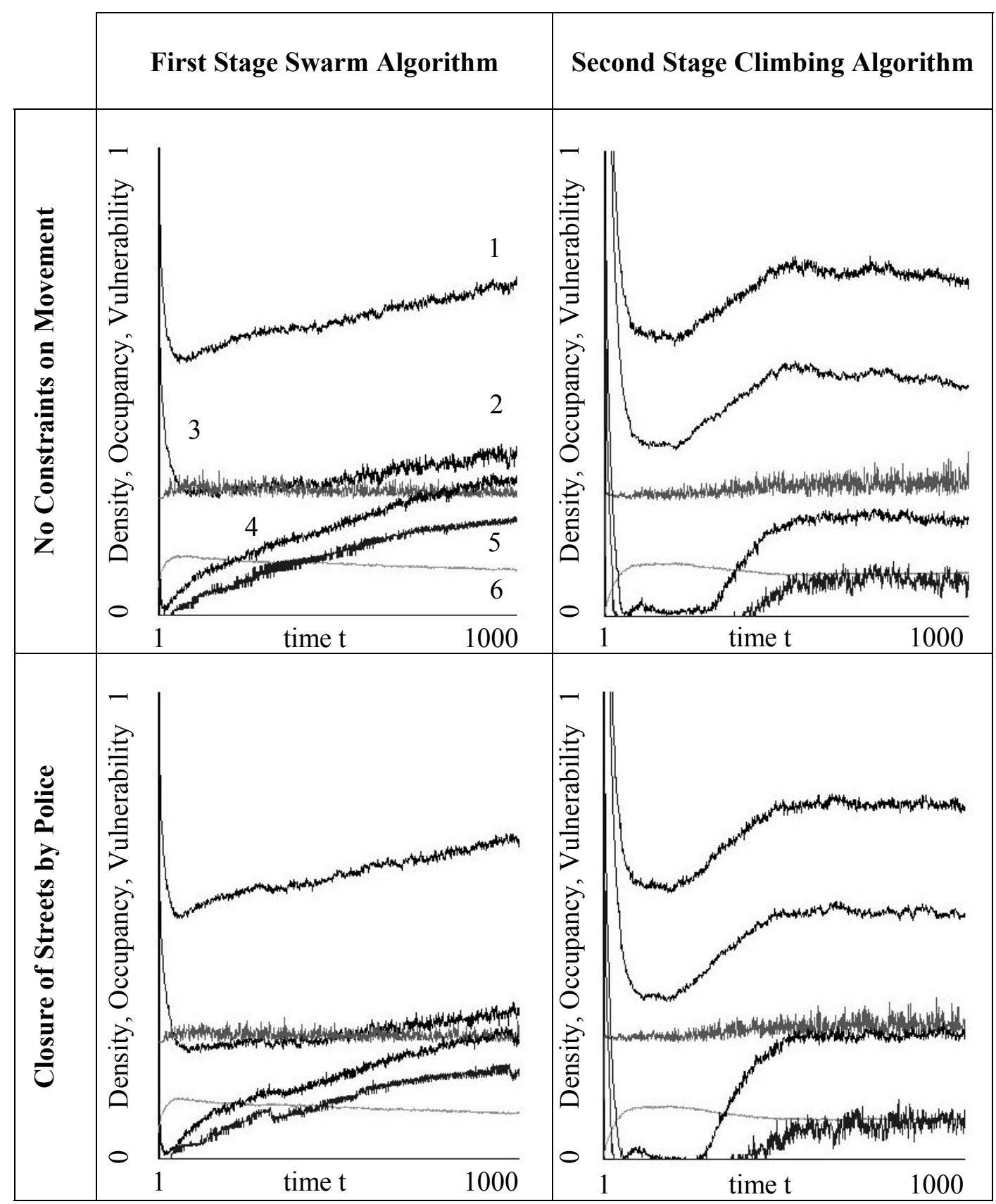

$\underline{1}$ Average walker density $\rho(t) / 3 \quad \underline{2}$ Average neighborhood walker density $\sigma(t) / 3$

$\underline{3}$ Average distance just traveled $U_{t+1} / 4 \quad \underline{4}$ Percent vulnerable $>0.5 \mathrm{ppm}^{2} Z_{t}(\Psi)>0.5$

$\underline{5}$ Percent vulnerable $>1 \mathrm{ppm}^{2} Z_{t}(\Psi)>1 \quad \underline{6}$ Percent Occupancy $\vartheta(t)$

Figure 9: Walker Densities and Safety Levels in the Two Stage Simulations

There was considerable variation in average densities, occupancies and vulnerabilities associated with the six new routes although in every case there was improvement. In general, the new routes which were longer in distance traveled and simpler in 
configuration spread the visitors over a wider area and this lowers the maximum and average densities of walkers. For example for the L-shaped route, the maximum density is $60 \%$ lower, the average neighborhood density is $45 \%$ lower and the average density is $36 \%$ lower than for the existing route. The interim solution adopted for the coming year (2002) which essentially breaks the loop by cutting the beginning from the end of the parade in the northern section, reduces crowding by $37 \%, 21 \%$ and $9 \%$ across these same measures and this is the least reduction of any of the six alternatives examined. These kinds of statistics form the basis of the evaluation of safety although there are many other measures from the model which are useful to the assessment. In future research, we will present these in more detail but for the moment, our concern is to outline the model and provide the rudiments of its use.

\section{Conclusions: Future Research}

Our current model is limited in its ability to simulate behavior across spatial scales and within different time periods due to software constraints on the number of agents handled and the level of resolution in terms of cell numbers. This means that we are unable to simulate a complete range of behaviors, which include panic situations where we need to represent the full agent population at very fine scales. As we move to full populations, we also require finer scales and finer time intervals which would enable us to represent speed and acceleration which are features of many pedestrian models (Helbing, 2001; Still, 2001). Only when we are able to represent all agents, would we be able to include behavioral protocols in the form of schedules governing movement and thus dispense with the rather crude way in which we model behavior as a response to various surfaces of accessibility. All these additions to the model require us to reprogram its structure in a more powerful language, thus enabling us to extend the number of agent types handled. This is work in progress. An important issue however is the need to develop software in which we can quickly visualize inputs and outputs from the model at different scales and through time. Some geographic information systems software can be extended in this way and we are already at work on using such software to handle visibility fields. It is unlikely 
however that in our new model, there will be anything other than a loose coupling to such software. We do not envisage embedding more powerful dynamic models of small-scale spatial events into GIS in the same way that more static urban models have been developed within standard software (Batty and Xie, 1994).

We also need much better data for such models. Path data is probably of lesser importance than attitudinal and related behavioral data that can only come through direct questionnaire. Our model depends on knowing the distribution of different types of agent and this in turn is reflected not only in their behaviors at the Carnival but also in various demographic and related characteristics. In short, following the law of requisite variety, we need to ensure that the richness of our models is matched by sufficiently rich data. This is clearly far from the case at present and although our concern for modeling small-scale events is being driven by new data sources as well as new methodologies, much better data sources are still required. This implies the expenditure of greater resources, notwithstanding the physical and ethical difficulties in actually obtaining the data that is required. To improve our models beyond what we have done here and in related work (Haklay, Thurstain-Goodwin, O'Sullivan and Schelhorn, 2001), data issues must be addressed.

Finally the model structure developed here provides a rather different perspective on the nature of control, design and planning. In many small-scale spatial event situations such as the movement of people into and out of high capacity building and vehicles, there are already major controls on what is possible, established by various legislative and local mandates. These must be built into the models directly and if they are to be altered in any way, then the experts and stakeholders who know most about these situations and what is possible must be intimately involved. This in turn requires the models to be accessible in a way that is not usually the case. This implies visualization but it also requires some form of translation into a context in which nonquantitative experts can interact with the process. In a sense, this is what has been attempted in participation within urban planning over the last 50 years or more but it is more than this. We need to establish environments in which a variety of stakeholders can be involved in the science and can provide essential inputs not only in the interpretation of results from the models but also the design of the models 
themselves. This presents a new frontier for geographic information science which the models developed here are just beginning to address.

\section{References}

BAER, A. E., 1974, A Simulation Model of Multidirectional Pedestrian Movement Within Physically Bounded Environments. Institute of Physical Planning, Report 47 (Pittsburgh, PA: Carnegie-Mellon University).

BATTY, M., 2001, Polynucleated urban landscapes. Urban Studies, 38, 635-655.

BATTY, M., and XIE, Y., 1994, Modeling inside GIS: Part 2: Selecting and calibrating urban models using ARC-INFO, International Journal of Geographical Information Systems, 8, 451-470.

BATTY, M., DODGE, M., JIANG, B., and SMITH, A., 1999, Geographical information systems and urban design. In Geographical Information and Planning, edited by J. Stillwell, S. Geertman, and S. Openshaw (Heidelberg, Germany: Springer), pp. 43-65.

BLUE, V. J. and ADLER, J. L., 2001, Cellular automata microsimulation for modeling bi-directional pedestrian walkways. Transportation Research B, 35, 292312 .

BONABEAU, E., DORIGO, M., and THERAULAZ, G., 1999, Swarm Intelligence: From Natural to Artificial Systems (New York: Oxford University Press).

BORGERS, A. and TIMMERMANS, H. A., 1986, A model of pedestrian route choice and demand for retail facilities within inner-city shopping areas. Geographical Analysis, 18, 115-128.

BOX, P., 2001, Spatial units as agents: making the landscape an equal player in agentbased simulations. In Integrating Geographic Information Systems and Agent-based Modeling Techniques, edited by H. Randy Gimblett (New York: Oxford University Press), pp. 59-82.

BURSTEDDE, C., KLAUCK, K., SCHADSCHNEIDER, A., and ZITTARZ, J., 2001, Simulation of pedestrian dynamics using a two-dimensional cellular automaton. Physica A, 295, 507-525.

CAMAZINE, S., DENEUBOURG, J-L., FRANKS, N. R., SNEYD, J., THERAULAZ, G. and BONABEAU, E., 2001, Self-Organization in Biological Systems (Princeton, NJ: Princeton University Press). 
CANETTI, E., 1962, Crowds and Power (London: Victor Gollancz).

CRG, 2001, Interim Report and Public Safety Profile Recommendations for 2001, (London: Carnival Review Group. Greater London Authority), available at http://www.london.gov.uk/mayor/carnival/interim_report/interim_review_report.pdf

DIJKSTRA, J., JESSURUN, J., and TIMMERMANS, H. J. P., 2002, A multi-agent cellular automata model of pedestrian movement. In Pedestrian and Evacuation Dynamics, edited by M. Schreckenberg and S. D. Sharma (Berlin: Springer-Verlag), pp. 173-180.

FRUIN, J. J., 1971, Pedestrian Planning and Design (New York: Metropolitan Association of Urban Designers and Environmental Planners, Inc.).

HAKLAY, M. THURSTAIN-GOODWIN, M. O'SULLIVAN, D. and SCHELHORN, T., 2001, "So go downtown": simulating pedestrian movement in town centers. Environment and Planning B, 28, 343-359.

HELBING, D., 1991, A mathematical model for the behavior of pedestrians. Behavioral Science, 36, 298-310.

HELBING, D., 2001, Traffic and related self-driven many-particle systems. Reviews of Modern Physics, 73, 1067-1141.

HELBING, D., FARKAS, I. J., MOLNAR, P., and VICSEK, T., 2001, Simulation of pedestrian crowds in normal and evacuation situations, In Pedestrian and Evacuation Dynamics, edited by M. Schreckenberg and S. D. Sharma (Berlin: Springer-Verlag), pp. 21-58.

HELBING, D., FARKAS, I., and VICSEK, T., 2000, Simulating dynamical features of escape panic. Nature, 407, 487-490.

HELBING, D., MOLNAR, P., FARKAS, I. J., and BOLAY, K., 2001, SelfOrganizing Pedestrian Movement, Environment and Planning B, 28, 361-383.

HELBING, D., SCHWEITZER, F., KELTSCH, J., and MOLNAR, P., 1997, Active walker model for the formation of animal and trail systems. Physical Review E, 56, 2527-2539.

HENDERSON, J. M., and QUANDT, R. E., 1980, Microeconomic Theory: A Mathematical Approach, $3^{\text {rd }}$ Edition (Tokyo: McGraw Hill).

HENDERSON, L. F., 1971, The statistics of crowd fluids. Nature, 229, 381-383.

HOOGENDOORN, S. P., BOVY, P. H. L., and DAAMEN, W., 2001, Microscopic pedestrian wayfinding and dynamics modeling. In Pedestrian and Evacuation Dynamics, edited by M. Schreckenberg and S. D. Sharma (Berlin: Springer-Verlag), pp. 123-154. 
IPS, 2002, Carnival Public Safety Project - Assessment of Route Design for the Notting Hill Carnival (London: Intelligent Space Partnership, Greater London Authority).

JOHNSON, S., 2001, Emergence: The Connected Lives of Ants, Brains, Cities, and Software (New York: Scribner).

KENNEDY, J., EBERHART, R. C., and SHUI, Y., 2001, Swarm Intelligence (San Francisco, CA: Morgan Kaufmann Publishers)

LOVAS, G. G., 1994, Modeling and simulation of pedestrian flow traffic. Transportation Research B, 28B, 429-443.

MARCH, L, and STEADMAN, P., 1971, The Geometry of Environment : An Introduction to Spatial Organization in Design (London: RIBA Publications).

NAGEL, K. and SCHRECKENBERG, M., 1992, A cellular automaton model for freeway traffic. Journal of Physique France, I2, 2221-2228.

PUSHKAREV, B. S., and ZUPAN, J. M., 1975, Urban Space for Pedestrians (Cambridge, MA: MIT Press).

RANNELLS, J., 1956, The Core of The City: A Pilot Study of Changing Land Uses in Central Business Districts (New York: Columbia University Press).

REYNOLDS, C. W., 1987, Flocks, herds, and schools: a distributed behavioral model. Computer Graphics, 21, 25-34.

SCHWEITZER, F., 1997, Active Brownian particles: artificial agents in physics. In Stochastic Dynamics, edited by L. Schimansky-Geier, and T. Poschel, Lecture Notes in Physics, Vol. 484 (Berlin: Springer), pp. 358-371.

SCHWEITZER, F., 1998, Modeling migration and economic agglomeration with active Brownian particles. Advances in Complex Systems, 1, 11-37.

SORNETTE, D., 2000, Critical Phenomena in Natural Sciences: Chaos, Fractals, Selforganization and Disorder (Berlin: Springer-Verlag).

STILL, G. K., 2001, Crowd Dynamics (PhD Thesis, Warwick, UK: University of Warwick), available at http://www.crowddynamics.com/

VICSEK, T., CZIROK, A. BEN-JACOB, E., COHEN, I., and SHOCHET, O., 1995, Novel type of phase transition in a systems of self-driven particles, Physical Review Letters, 75, 1226-1229. 